\title{
Three-Dimensional Piezothermoelastic Stress of a Finite Functionally Graded Cylindrical Shell with Piezoelectric Layer
}

\author{
Yong-gang Kang, Zhong-qi Wang, and Gongnan Xie \\ The Key Laboratory of Contemporary Design and Integrated Manufacturing Technology, Ministry of Education, \\ Northwestern Polytechnical University, P.O. Box 409, Xi'An, Shaanxi 710072, China
}

Correspondence should be addressed to Yong-gang Kang; kangyonggang@nwpu.edu.cn

Received 27 September 2013; Accepted 27 October 2013

Academic Editor: Manyu Xiao

Copyright (c) 2013 Yong-gang Kang et al. This is an open access article distributed under the Creative Commons Attribution License, which permits unrestricted use, distribution, and reproduction in any medium, provided the original work is properly cited.

Three-dimensional piezothermoelastic solutions for a finite functionally graded cylindrical shell with piezoelectric layer are carried out in this paper. The cylindrical shell is simply supported at four end edges and is subjected to axisymmetric thermomechanical loads. The piezoelectric layers are polarized along radial direction as a sensor. The material properties are assumed to be temperature independent and radially dependent but are assumed to be homogeneous in each layer; the variables are expanded in Fourier series to satisfy the boundary conditions and multilayer approach is used. Numerical results of mullite/molybdenum functionally graded cylindrical shell are presented; the temperature change, stresses, electric potential, and electric displacement distributions are given and briefly discussed.

\section{Introduction}

Functionally graded materials (FGMs) are new kind of inhomogeneous composite materials with continuously varying properties. The spatial grading in composition and microstructure of FGMs can be adjusted in order to obtain specific properties or to reduce the magnitude of mechanical and thermal stresses. Piezoelectric materials are probably the most popular active materials which act as sensors and actuators and have been used widely in structural health monitoring, vibration, and noise control as well as many other areas. Among these, piezoelectric bimorph and multimorph are commonly employed. Usually, these structures are made of two or more layers of piezoelectric sheets and are jointed by bonding agents. However, such laminated piezoelectric structures suffer from high stress concentration near the interface due to the abrupt changes in both material composition and thermoelectroelastic properties, which can cause severe deterioration of the bonding layer strength and reduce the lifetime of the structures. To overcome the drawbacks and meet some particular requirements for performance and reliability, the functionally graded materials (FGMs) and piezoelectric material are used together, which called functionally graded piezoelectric materials (FGPMs). A new type of structure was developed.

The fabrication and property investigation of FGPMs have attracted great attention from the research community. Among the early investigators, PintoCarreia et al. [1] derived and solved the coupled displacement and electrical field equations for a piezoelectric cylindrical shell based on the third-order shear deformation theory and the finite element method. Using classical laminate theory, the piezothermoelastic behavior of a piezoelectric composite actuator with functionally graded microstructure was analyzed [2]. Ootao and Tanigawa [3-7] exactly analyzed the transient piezothermoelastic problem of a functionally graded hollow sphere, a simply supported cylindrical composite panel which composed of crossply or angleply and piezoelectric layer under the state of generalized plane deformation, a rectangular composite plate composed of cross-ply laminate and piezoelectric material, and a functionally graded rectangular plate bonded to a piezoelectric plate due to partial heat supply. Using the power series expansion method, Chen and his coworkers $[8,9]$ successfully derived two-dimensional exact elasticity solution and studied both the direct and inverse piezoelectric effects of the piezothermoelastic behavior of 
circular laminated piezoelectric cylindrical shells and functionally graded piezoelectric cylindrical shells subjected to axisymmetric loading. Kapuria and Achary [10] presented a new coupled consistent third-order theory for static thermoelectromechanical response of hybrid piezoelectric laminated plates. Shao [11] presented solutions for temperature, displacement and stress fields in an FG hollow cylinder using a multilayered approach based on the laminated composite theory. Huang et al. [12] derived the analytical solutions for FGP beams under both mechanical and electrical loads from the two-dimensional equations of piezoelectricity, in which the elastic, piezoelectric and dielectric coefficients of the piezoelectric beams were assumed to vary along the beam thickness direction only. Yang and Xiang [13] used the Timoshenko beam theory to investigate the static bending and dynamic response of FGP actuators under combined thermalelectromechanical loading. Ying and Wang [14] employed the separation of variable technique to carry out twodimensional elastic-dynamic analysis of simply supported hollow cylinders of finite length excited by nonuniform thermal shock. Reference [15] proposed improved layered shell finite element solution for the coupled thermoelectromechanical responses of smart fiber reinforced composite shell panels under piezothermoelastic loading and showed that piezoelectric effect has a significant influence on the response of such shells. The bending behavior of a circularly curved FGP cantilever actuator under an applied electrical load and heat conduction was investigated [16]. Infinitesimal axisymmetric deformation of a functionally graded shell with piezoelectric layers perfectly bonded to its inner and outer surfaces subjected to thermo-electro-mechanical loads was studied by Alibeigloo [17]. Alashti and Khorsand [18] carried out three-dimensional static analyses of FG cylindrical shells with piezoelectric layers under the effect of thermoelectromechanical loads, using the differential quadrature method.

In this paper, a functionally graded cylindrical shell with piezoelectric layer is investigated. The thermal and mechanical loads are applied on the cylinder asymmetrically. In order to obtain analytical solutions for temperature, displacements, and stresses for the three-dimensional piezothermoelastic problem, it is assumed that the piezoelectric functionally graded cylindrical shell is composed of $N$ fictitious layers and one piezoelectric layer, and each layer of the functionally graded material is homogeneous and isotropic.

\section{Basic Equations}

A piezoelectric functionally graded cylindrical shell with finite length $l$, internal radius $r_{a}$, external radius $r_{b}$, and circumferential angle $\theta_{0}$ is considered (as shown in Figure 1), and it is assumed that the piezoelectric layer is perfectly bonded, the inner radius of piezoelectric layer is $r_{c}$, and the radius of the interface between the $i$ th and $(i+1)$ th layer is $r_{i}$. Cylindrical coordinates $r, \theta$, and $z$ are used in analysis. This functionally graded cylindrical shell is simply supported at its four end edges and subjected to nonuniform steady-state thermal loads $T_{a}(\theta, z)$ and $T_{b}(\theta, z)$ on the inner and outer surface. The temperatures at end edges keep zero. Nonuniform pressure loadings $q_{a}(\theta, z)$ and $q_{b}(\theta, z)$ are applied to the

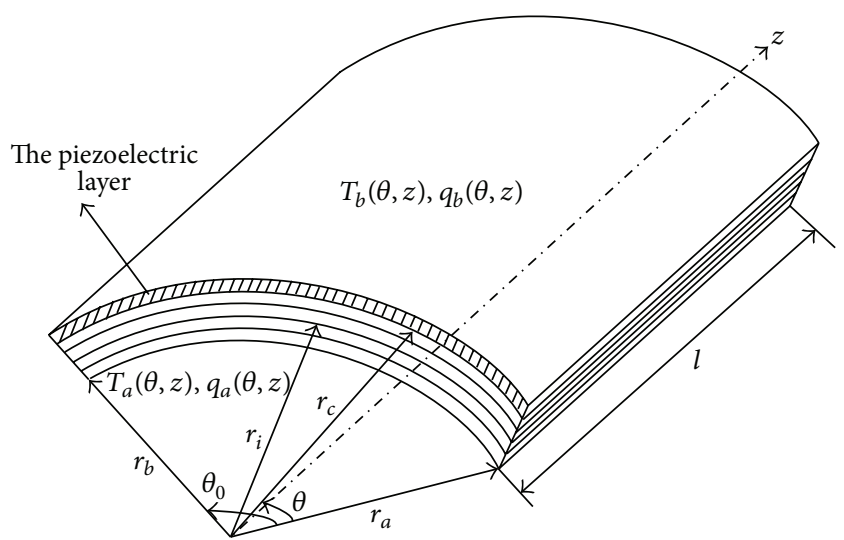

FIgURE 1: A piezoelectric functionally graded cylindrical shell.

inner and outer surfaces of the panel, respectively. Details of piezoelectric functionally graded cylindrical shell are shown in Figure 1.

It is assumed that the piezoelectric functionally graded cylindrical shell is composed of $N$ fictitious layers and one piezoelectric layer, and each layer of the functionally graded material is homogeneous and isotropic. The law of mixtures proposed by Kerner, which was derived based on the assumption that there is a granular phase embedded in a matrix phase, is applied to describe the variations of material properties in the radial direction of the functionally graded cylinder shell. Young's modulus $E$, Poisson's ratio $\mu$, thermal conductivity coefficient $\lambda$, and thermal expansion coefficient $\alpha$ can be expressed as

$$
\begin{aligned}
& E(r)=V_{m} E_{m}+\left(1-V_{m}\right) E_{C} \\
& \mu(r)=V_{m} \mu_{m}+\left(1-V_{m}\right) \mu_{C} \\
& \lambda(r)=V_{m} \lambda_{m}+\left(1-V_{m}\right) \lambda_{C} \\
& \alpha(r)=V_{m} \alpha_{m}+\left(1-V_{m}\right) \alpha_{C}
\end{aligned}
$$

where the subscripts $m$ and $c$ represent the metal and ceramic, respectively. $V_{m}$ is the volume fraction in the functionally graded material:

$$
V_{m}(r)=\left(\frac{r-r_{a}}{r_{b}-r_{a}}\right)^{p} .
$$

Here, $p$ is a constant material. The effects of the constant $p$ on the temperature, displacements, and thermal stresses are fully discussed in $[6,19]$. For the sake of brevity, discussion of these effects is omitted here.

The basic piezothermoelastic equations for the $i$ th layer can be expressed as follows.

2.1. Conduction Equations and Equilibrium Equations. The heat conduction equation can be expressed as

$$
\left(\frac{\partial^{2}}{\partial r^{2}}+\frac{1}{r} \frac{\partial}{\partial r}+\frac{1}{r^{2}} \frac{\partial^{2}}{\partial \theta^{2}}+\frac{\partial^{2}}{\partial z^{2}}\right) T_{i}=0
$$


where $\bar{T}_{i}$ is the temperature in the $i$ th layer. While the equilibrium equation of the piezoelectric functionally graded cylindrical shell is

$$
\begin{gathered}
\frac{\partial \sigma_{r r i}}{\partial r}+\frac{1}{r} \frac{\partial \sigma_{r \theta i}}{\partial \theta}+\frac{\partial \sigma_{r z i}}{\partial z}+\frac{\sigma_{r r i}+\sigma_{\theta \theta i}}{r}=0, \\
\frac{\partial \sigma_{r \theta i}}{\partial r}+\frac{1}{r} \frac{\partial \sigma_{\theta \theta i}}{\partial \theta}+\frac{\partial \sigma_{\theta z i}}{\partial z}+\frac{2 \sigma_{r \theta i}}{r}=0 \\
\frac{\partial \sigma_{r z i}}{\partial r}+\frac{1}{r} \frac{\partial \sigma_{\theta z i}}{\partial \theta}+\frac{\partial \sigma_{z z i}}{\partial z}+\frac{\sigma_{r z i}}{r}=0
\end{gathered}
$$

the electrostatics equation can be written as

$$
\frac{\partial D_{r}}{\partial r}+\frac{D_{r}}{r}+\frac{1}{r} \frac{\partial D_{\theta}}{\partial \theta}+\frac{\partial D_{z}}{\partial z}=0
$$

The Constitutive equation can be

$$
\begin{aligned}
\left\{\begin{array}{c}
\sigma_{r r i} \\
\sigma_{\theta \theta i} \\
\sigma_{z z i} \\
\sigma_{\theta z i} \\
\sigma_{r z i} \\
\sigma_{r \theta i}
\end{array}\right\}= & {\left[\begin{array}{cccccc}
C_{11 i} & C_{12 i} & C_{13 i} & 0 & 0 & 0 \\
C_{21 i} & C_{22 i} & C_{23 i} & 0 & 0 & 0 \\
C_{13 i} & C_{23 i} & C_{33 i} & 0 & 0 & 0 \\
0 & 0 & 0 & C_{44 i} & 0 & 0 \\
0 & 0 & 0 & 0 & C_{55 i} & 0 \\
0 & 0 & 0 & 0 & 0 & C_{66 i}
\end{array}\right]\left\{\begin{array}{l}
\varepsilon_{r r i} \\
\varepsilon_{\theta \theta i} \\
\varepsilon_{z z i} \\
\varepsilon_{\theta z i} \\
\varepsilon_{r z i} \\
\varepsilon_{r \theta i}
\end{array}\right\} } \\
& -\left\{\begin{array}{c}
\beta_{r i} T_{i} \\
\beta_{\theta i} T_{i} \\
\beta_{z i} T_{i} \\
0 \\
0 \\
0
\end{array}\right\}-\left(\begin{array}{ccc}
e_{1} & 0 & 0 \\
e_{2} & 0 & 0 \\
e_{3} & 0 & 0 \\
0 & 0 & 0 \\
0 & 0 & e_{5} \\
0 & e_{6} & 0
\end{array}\right)\left\{\begin{array}{l}
E_{r} \\
E_{\theta} \\
E_{z}
\end{array}\right\},
\end{aligned}
$$

where

$$
\begin{array}{r}
\beta_{r i}=\left(C_{11 i}+C_{12 i}+C_{13 i}\right) \alpha_{i} \\
\beta_{\theta i}=\left(C_{12 i}+C_{22 i}+C_{23 i}\right) \alpha_{i} \\
\beta_{z i}=\left(C_{13 i}+C_{23 i}+C_{33 i}\right) \alpha_{i} \\
\quad i=1,2,3, \ldots, N+1,
\end{array}
$$

In the case of the stress-strain relations of functionally graded cylindrical shell $(i=1$ to $N)$, the terms $e_{k}$ and $E_{k}$ on the right side in (5) vanished. And $C_{k l i}, \beta$ are elastic constants and thermoelastic coefficients.

The constitutive equations for the electric field are

$$
\begin{gathered}
D_{r}=e_{1} \varepsilon_{r r}+e_{2} \varepsilon_{\theta \theta}+e_{3} \varepsilon_{z z}+\eta_{1} E_{r}+P_{1} T_{1}, \\
D_{\theta}=e_{6} \varepsilon_{r \theta}+\eta_{2} E_{\theta}, \\
D_{z}=e_{5} \varepsilon_{r z}+\eta_{3} E_{z},
\end{gathered}
$$

$$
\begin{gathered}
\varepsilon_{r r i}=\frac{\partial u_{i}}{\partial r} ; \quad \varepsilon_{\theta \theta i}=\frac{1}{r}\left(\frac{\partial v_{i}}{\partial \theta}+u_{i}\right) ; \\
\varepsilon_{z z i}=\frac{\partial w_{i}}{\partial z} ; \quad \varepsilon_{r \theta i}=\frac{1}{r} \frac{\partial u_{i}}{\partial \theta}+\frac{\partial v_{i}}{\partial r}-\frac{\partial v_{i}}{r} ; \\
\varepsilon_{\theta z i}=\frac{\partial v_{i}}{\partial z}+\frac{1}{r} \frac{\partial w_{i}}{\partial \theta} ; \quad \varepsilon_{z r i}=\frac{\partial u_{i}}{\partial z}+\frac{\partial w_{i}}{\partial r} ; \\
E_{r}=-\frac{\partial \phi}{\partial r} ; \quad E_{\theta}=-\frac{1}{r} \frac{\partial \phi}{\partial \theta} ; \quad i=1,2,3, \ldots, N+1 .
\end{gathered}
$$

2.2. Boundary and Continuity Conditions. Consider the following:

$$
\begin{gathered}
\theta=0, \quad \theta_{0}: T_{i}=0 \\
\bar{z}=0, \quad \frac{l}{r_{0}}: T_{i}=0 \\
\bar{r}=\frac{r_{1}}{r_{0}}: T_{1}=T_{a}(\theta, \bar{z}) \\
\bar{r}=\frac{r_{N+2}}{r_{0}}: T_{N+1}=T_{b}(\theta, \bar{z}) \\
\bar{r}=\frac{r_{i+1}}{r_{0}}: T_{i}=T_{i+1} ; \\
\lambda_{i} \frac{\partial T_{i}}{\partial r}=\lambda_{i+1} \frac{\partial T_{i+1}}{\partial r}, \quad i=1,2,3, \ldots, N,
\end{gathered}
$$

where $\lambda_{i}$ and $\lambda_{i+1}$ are the thermal dimensionless conductivity parameter in $i$ th and $i+1$ th layer, respectively. $T_{a}(\theta, z)$ and $T_{b}(\theta, z)$ are temperatures in the inner and outer surfaces.

For the simply supported panel, we assumed that the edges of the piezoelectric layer are electrically grounded, so the boundary conditions and continuous conditions between layers of displacements and stresses can be expressed as

$$
\begin{gathered}
\theta=0, \quad \theta_{0}: u_{i}=w_{i}=0 ; \quad \sigma_{\theta \theta i}=\sigma_{r \theta i}=\sigma_{\theta z i}=0 ; \\
\phi=0, \quad i=1,2,3, \ldots, N+1, \\
z=0, \quad \frac{l}{r_{0}}: u_{i}=v_{i}=0 ; \quad \sigma_{z z i}=\sigma_{r z i}=\sigma_{\theta z i}=0 ; \\
\phi=0, \quad i=1,2,3, \ldots, N+1,
\end{gathered}
$$

$$
\begin{gathered}
r=\frac{r_{1}}{r_{0}}: \sigma_{r, 1}=q_{a}(\theta, z) ; \quad \sigma_{r z, 1}=\sigma_{r \theta, 1}=0 \\
r=\frac{r_{N+1}}{r_{0}}: \phi=0,
\end{gathered}
$$

$$
\begin{gathered}
r=\frac{r_{N+2}}{r_{0}}: D_{r}=0 ; \quad \sigma_{r \theta, N}=\sigma_{r z, N}=0 ; \quad \sigma_{r r, N+1}=q_{b}(\theta, z) \\
r=\frac{r_{i}}{r_{0}}: \sigma_{r r i}=\sigma_{r r, i+1} ; \quad \sigma_{r \theta i}=\sigma_{r \theta, i+1} ; \quad \sigma_{r z i}=\sigma_{r z, i+1} ;
\end{gathered}
$$




$$
\begin{array}{r}
u_{i}=u_{i+1} ; \quad v_{i}=v_{i+1} ; \quad w_{i}=w_{i+1} \\
i=1,2,3, \ldots, N+1+1 .
\end{array}
$$

To simplify the solving process of the $3 \mathrm{D}$ piezothermomechanical problem, the following dimensionless variables are introduced:

$$
\begin{gathered}
\bar{r}=\frac{r}{r_{0}}, \quad \bar{z}=\frac{z}{r_{0}}, \quad \bar{L}=\frac{l}{r_{0}}, \quad \overline{\alpha_{k}}=\frac{\alpha_{k}}{\alpha_{0}}, \\
\overline{\lambda_{k}}=\frac{\lambda_{k}}{\lambda_{0}}, \quad \overline{k_{k}}=\frac{k_{k}}{k_{0}}, \quad \overline{T_{k}}=\frac{T}{T_{0}}, \quad \overline{T_{a}}=\frac{T_{a}}{T_{0}}, \\
\overline{T_{b}}=\frac{T_{b}}{T_{0}}, \quad \bar{q}_{a}=\frac{q_{a}}{\alpha_{0} Y_{0} T_{0}}, \quad \bar{q}_{b}=\frac{q_{b}}{\alpha_{0} Y_{0} T_{0}}, \\
\bar{\sigma}_{k l i}=\frac{\sigma_{k l i}}{\alpha_{0} Y_{0} T_{0}}, \quad \bar{\varepsilon}_{k l i}=\frac{\varepsilon_{k l i}}{\alpha_{0} T_{0}}, \quad\left\{\bar{u}_{i}, \bar{v}_{i}, \bar{w}_{i}\right\}=\frac{\left\{u_{i}, v_{i}, w_{i}\right\}}{\alpha_{0} r_{0} T_{0}} \\
\bar{\alpha}_{k i}=\frac{\alpha_{k i}}{\alpha_{0}}, \quad \bar{C}_{k l i}=\frac{C_{k l i}}{Y_{0}}, \quad \bar{E}_{k}=\frac{E_{k}\left|d_{1}\right|}{\alpha_{0} T_{0}} ; \\
\bar{d}_{k}=\frac{D_{k}}{\alpha_{0} T_{0} Y_{0}\left|d_{1}\right|}, \quad \bar{\phi}_{k}=\frac{\phi\left|d_{1}\right|}{\alpha_{0} T_{0} r_{0}}, \\
\frac{e_{k}}{\bar{e}_{k}\left|d_{1}\right|}, \quad \bar{\eta}_{k}=\frac{\eta_{k}}{Y_{0}\left|d_{1}\right|^{2}}, \quad \bar{P}_{1}=\frac{P_{1}}{\alpha_{0} Y_{0}\left|d_{1}\right|}
\end{gathered}
$$

where $r_{0}$ is reference value of radius and $Y_{0}, \alpha_{0}, k_{0}, \lambda_{0}$ and $T_{0}$ are reference values of Young's modulus, thermal expansion coefficient, thermal diffusion coefficient, thermal conductivity coefficient, and temperature, respectively, where $\sigma_{k l, i}$ are the stress components, $\varepsilon_{k l, i}$ are the strain components, $\left(u_{i}, v_{i}, w_{i}\right)$ are the displacement components, $C_{k l, i}$ are the elastic stiffness constants, $D_{k}$ are the electric displacement components, $e_{k}$ are the piezoelectric coefficients, $\eta_{k}$ are the dielectric constants, $P_{1}$ is the pyroelectric constant, and $d_{1}$ is the piezoelectric modulus.

The dimensionless displacement-expressed equilibrium equations can be expressed as

$$
\begin{aligned}
& {\left[\bar{C}_{11 i}\left(\frac{\partial^{2}}{\partial \bar{r}^{2}}+\frac{1}{\bar{r}} \frac{\partial}{\partial \bar{r}}\right)-\frac{\bar{C}_{66 i} \partial^{2}}{\bar{r}^{2} \partial \theta}+\bar{C}_{55 i} \frac{\partial^{2}}{\partial \bar{z}^{2}}-\frac{\bar{C}_{22 i}}{\bar{r}^{2}}\right] u_{i}} \\
& +\frac{1}{\bar{r}}\left(\bar{C}_{12 i}+\bar{C}_{66 i}\right) \frac{\partial^{2} \bar{v}_{i}}{\partial \bar{r} \partial \theta} \\
& +\frac{1}{\bar{r}^{2}}\left(\bar{C}_{22 i}+\bar{C}_{66 i}\right) \frac{\partial \bar{v}_{i}}{\partial \theta}+\left(\bar{C}_{13 i}+\bar{C}_{55 i}\right) \frac{\partial^{2} \bar{w}_{i}}{\partial \bar{r} \partial \bar{z}} \\
& +\left(\bar{C}_{13 i}-\bar{C}_{23 i}\right) \frac{1}{\bar{r}} \frac{\partial \bar{w}_{i}}{\partial \bar{z}}-\bar{\beta}_{r i} \frac{\partial \bar{T}_{i}}{\partial \bar{r}} \\
& \quad-\left(\bar{\beta}_{r i}-\bar{\beta}_{\theta i}\right) \frac{\bar{T}_{i}}{\bar{r}}+\bar{e}_{1} \frac{\partial^{2} \bar{\phi}}{\partial \bar{r}^{2}}+\bar{e}_{6} \frac{\partial^{2} \bar{\phi}}{\bar{r}^{2} \partial \theta^{2}} \\
& +\bar{e}_{5} \frac{\partial^{2} \bar{\phi}}{\partial \bar{z}^{2}}+\left(\bar{e}_{1}-\bar{e}_{2}\right) \frac{1}{\bar{r}} \frac{\partial \bar{\phi}}{\partial \bar{r}}=0,
\end{aligned}
$$

$$
\begin{aligned}
& \left(\bar{C}_{12 i}+\bar{C}_{66 i}\right) \frac{1}{\bar{r}} \frac{\partial^{2} \bar{u}_{i}}{\partial \bar{r} \partial \theta}+\left(\bar{C}_{22 i}+\bar{C}_{66 i}\right) \frac{1}{\bar{r}^{2}} \frac{\partial \bar{u}_{i}}{\partial \theta} \\
& +\bar{C}_{66 i}\left(\frac{\partial^{2} \bar{v}_{i}}{\partial \bar{r}^{2}}+\frac{1}{\bar{r}} \frac{\partial \bar{v}_{i}}{\partial \bar{r}}-\frac{\bar{v}_{i}}{\bar{r}^{2}}\right)+\bar{C}_{22 i} \frac{1}{\bar{r}^{2}} \frac{\partial^{2} \bar{v}_{i}}{\partial \theta^{2}} \\
& +\bar{C}_{44 i} \frac{\partial^{2} \bar{v}_{i}}{\partial \bar{z}^{2}}+\left(\bar{C}_{23 i}+\bar{C}_{44 i}\right) \frac{1}{\bar{r}} \frac{\partial^{2} \bar{w}_{i}}{\partial \theta \partial \bar{z}} \\
& -\bar{\beta}_{\theta i} \frac{1}{\bar{r}} \frac{\partial \bar{T}_{i}}{\partial \theta}+\left(\bar{e}_{2}+\bar{e}_{6}\right) \frac{1}{\bar{r}} \frac{\partial^{2} \bar{\phi}}{\partial \bar{r} \partial \theta}=0 \\
& \left(\bar{C}_{13 i}+\bar{C}_{55 i}\right) \frac{\partial^{2} \bar{u}_{i}}{\partial \bar{r} \partial \bar{z}}+\left(\bar{C}_{23 i}+\bar{C}_{55 i}\right) \frac{1}{\bar{r}} \frac{\partial \bar{u}_{i}}{\partial \bar{z}} \\
& +\left(\bar{C}_{23 i}+\bar{C}_{44 i}\right) \frac{1}{\bar{r}} \frac{\partial^{2} \bar{v}_{i}}{\partial \theta \partial \bar{z}}+\bar{C}_{55 i} \frac{\partial^{2} \bar{w}_{i}}{\partial \bar{r}^{2}} \\
& +\bar{C}_{55 i} \frac{1}{\bar{r}} \frac{\partial \bar{w}_{i}}{\partial \bar{r}}+\bar{C}_{44 i} \frac{\partial^{2} \bar{w}_{i}}{\bar{r}^{2} \partial \theta^{2}}+\bar{C}_{33 i} \frac{\partial^{2} \bar{w}_{i}}{\partial \bar{z}^{2}} \\
& +\bar{\beta}_{\bar{z} i} \frac{\partial \bar{T}_{i}}{\partial \bar{z}}+\left(\bar{e}_{3}+\bar{e}_{6}\right) \frac{\partial^{2} \bar{\phi}}{\partial \bar{r} \partial \bar{z}}+\bar{e}_{5} \frac{1}{\bar{r}} \frac{\partial \bar{\phi}}{\partial \bar{z}}=0
\end{aligned}
$$

The dimensionless displacement-expressed electrostatic equation can be expressed as

$$
\begin{gathered}
\bar{e}_{1} \frac{\partial^{2} \bar{u}_{i}}{\partial \bar{r}^{2}}+\left(\bar{e}_{1}+\bar{e}_{2}\right) \frac{1}{\bar{r}} \frac{\partial \bar{u}_{i}}{\partial \bar{r}}+\bar{e}_{6} \frac{1}{\bar{r}^{2}} \frac{\partial^{2} \bar{u}_{i}}{\partial \theta^{2}}+\bar{e}_{5} \frac{\partial^{2} \bar{u}_{i}}{\partial \bar{z}^{2}} \\
+\left(\bar{e}_{2}+\bar{e}_{6}\right) \frac{1}{\bar{r}} \frac{\partial^{2} \bar{v}_{i}}{\partial r \partial \theta}-\bar{e}_{6} \frac{1}{\bar{r}^{2}} \frac{\partial \bar{v}_{i}}{\partial \theta} \\
+\left(\bar{e}_{3}+\bar{e}_{5}\right) \frac{\partial^{2} \bar{w}_{i}}{\partial \bar{r} \partial \bar{z}}+\bar{e}_{3} \frac{1}{\bar{r}} \frac{\partial \bar{w}_{i}}{\partial \bar{z}}-\bar{\eta}_{1}\left(\frac{\partial^{2} \bar{\phi}}{\partial \bar{r}^{2}}+\frac{1}{\bar{r}} \frac{\partial \bar{\phi}}{\partial \bar{r}}\right) \\
-\bar{\eta}_{2} \frac{1}{\bar{r}^{2}} \frac{\partial^{2} \bar{\phi}}{\partial \bar{\theta}^{2}}-\bar{\eta}_{3} \frac{\partial^{2} \bar{\phi}}{\partial \bar{z}^{2}}+\bar{p}_{1}\left(\frac{\partial \bar{T}_{i}}{\partial \bar{r}}+\frac{1}{\bar{r}} \bar{T}_{i}\right)=0 .
\end{gathered}
$$

Using Navier trigonometric series, solutions of (3), (13a) to (13c) and (14) which satisfy the ends displacements boundary conditions (11a) to (11b), (14) can be written as

$$
\begin{array}{r}
\bar{T}_{i}(\bar{r}, \theta \cdot \bar{z})=\sum_{n=1}^{\infty} \sum_{m=1}^{\infty} \bar{T}_{n m i}(\bar{r}) \sin (a \theta) \sin (b \bar{z}) \\
i=1,2,3, \ldots, N+1, \\
\overline{u_{i}}(\bar{r}, \theta \cdot \bar{z})=\sum_{n=1}^{\infty} \sum_{m=1}^{\infty} \bar{u}_{n m i}(\bar{r}) \sin (a \theta) \sin (b \bar{z}) \\
i=1,2,3, \ldots, N+1, \\
\overline{v_{i}}(\bar{r}, \theta \cdot \bar{z})=\sum_{n=1}^{\infty} \sum_{m=1}^{\infty} \bar{v}_{n m i}(\bar{r}) \cos (a \theta) \sin (b \bar{z}) \\
i=1,2,3, \ldots, N+1,
\end{array}
$$




$$
\begin{array}{r}
\overline{w_{i}}(\bar{r}, \theta \cdot \bar{z})=\sum_{n=1}^{\infty} \sum_{m=1}^{\infty} \bar{w}_{n m i}(\bar{r}) \sin (a \theta) \cos (b \bar{z}) \\
i=1,2,3, \ldots, N+1, \\
\overline{\phi_{i}}(\bar{r}, \theta \cdot \bar{z})=\sum_{n=1}^{\infty} \sum_{m=1}^{\infty} \bar{\phi}_{n m i}(\bar{r}) \sin (a \theta) \sin (b \bar{z}) \\
i=N+1,
\end{array}
$$

where $a=m \pi / \theta^{0}, b=n \pi / L ; \bar{T}_{n m i}, \bar{u}_{n m i}, \bar{v}_{n m i}, \bar{w}_{n m i}$, and $\bar{\phi}_{n m i}$ are unknown functions, Substituting trigonometric series (15a) to (15e) into (3) and (13a) to (13c) and (14), we can obtain

$$
\begin{aligned}
& \frac{d^{2} \bar{T}_{n m i}}{d \bar{r}^{2}}+\frac{1}{\bar{r}} \frac{d \bar{T}_{n m i}}{d \bar{r}}-\frac{a^{2}}{\bar{r}^{2}} \bar{T}_{n m i}-b^{2} \bar{T}_{n m i}=0, \\
& i=1,2,3, \ldots, N+1, \\
& {\left[\bar{C}_{11 i}\left(\frac{d^{2}}{d \bar{r}^{2}}+\frac{1}{\bar{r}} \frac{d}{d \bar{r}}\right)-a^{2} \bar{C}_{66 i} \frac{1}{\bar{r}^{2}}-b^{2} \bar{C}_{55 i}-\bar{C}_{22 i} \frac{1}{\bar{r}^{2}}\right] \bar{u}_{n m i}} \\
& -a\left(\bar{C}_{12 i}+\bar{C}_{66 i}\right) \frac{1}{\bar{r}} \frac{d \bar{v}_{n m i}}{d \bar{r}}+a\left(\bar{C}_{22 i}+\bar{C}_{66 i}\right) \frac{1}{\bar{r}^{2}} \bar{v}_{n m i} \\
& -b\left[\left(\bar{C}_{13 i}+\bar{C}_{55 i}\right) \frac{d}{d \bar{r}}+\left(\bar{C}_{13 i}-\bar{C}_{23 i}\right) \frac{1}{\bar{r}}\right] \bar{w}_{n m i}-\bar{\beta}_{r i} \frac{d \bar{T}_{n m i}}{d \bar{r}} \\
& -\left(\bar{\beta}_{r i}-\bar{\beta}_{\theta i}\right) \frac{\bar{T}_{n m i}}{\bar{r}}+\bar{e}_{1} \frac{d^{2} \bar{\phi}_{n m i}}{d \bar{r}^{2}}-a^{2} \bar{e}_{6} \frac{1}{\bar{r}^{2}} \bar{\phi}_{n m i} \\
& -b^{2} \bar{e}_{5} \bar{\phi}_{n m i}+\left(\bar{e}_{1}-\bar{e}_{2}\right) \frac{1}{\bar{r}} \frac{d \bar{\phi}_{n m i}}{d \bar{r}}=0 \text {, } \\
& a\left[\left(\bar{C}_{12 i}+\bar{C}_{66 i}\right) \frac{1}{\bar{r}} \frac{d}{d \bar{r}}+\left(\bar{C}_{22 i}+\bar{C}_{66 i}\right) \frac{1}{\bar{r}^{2}}\right] \bar{u}_{n m i} \\
& +\bar{C}_{66 i}\left(\frac{d^{2}}{d \bar{r}^{2}}+\frac{1}{\bar{r}} \frac{d}{d \bar{r}}-\frac{1}{\bar{r}^{2}}\right) \bar{v}_{n m i}-a^{2} \bar{C}_{22 i} \frac{1}{\bar{r}^{2}} \bar{v}_{n m i} \\
& -b^{2} \bar{C}_{44 i} \bar{v}_{n m i}-a b\left(\bar{C}_{23 i}+\bar{C}_{44 i}\right) \frac{1}{\bar{r}} \bar{w}_{n m i} \\
& -a \bar{\beta}_{\theta i} \frac{1}{\bar{r}} \bar{T}_{n m i}+a\left(\bar{e}_{2}+\bar{e}_{6}\right) \frac{1}{\bar{r}} \bar{\phi}_{n m i}=0, \\
& b\left[\left(\bar{C}_{13 i}+\bar{C}_{55 i}\right) \frac{d}{d \bar{r}}+\left(\bar{C}_{23 i}+\bar{C}_{55 i}\right) \frac{1}{\bar{r}}\right] \bar{u}_{n m i} \\
& -a b\left(\bar{C}_{23 i}+\bar{C}_{44 i}\right) \frac{1}{\bar{r}} \bar{v}_{n m i}+\bar{C}_{55 i} \frac{d^{2} \bar{w}_{n m i}}{d \bar{r}^{2}} \\
& +\left(\bar{C}_{55 i} \frac{1}{\bar{r}} \frac{d}{d \bar{r}}-\alpha^{2} \bar{C}_{44 i} \frac{1}{\bar{r}^{2}}-b^{2} \bar{C}_{33 i}\right) \bar{w}_{n m i} \\
& -b \bar{\beta}_{\bar{z} i} \bar{T}_{n m i}+b\left[\left(\bar{e}_{3}+\bar{e}_{6}\right) \frac{d}{d \bar{r}}+\bar{e}_{5} \frac{1}{\bar{r}}\right] \bar{\phi}_{n m i}=0,
\end{aligned}
$$

$$
\begin{gathered}
{\left[\bar{e}_{1} \frac{d^{2}}{d \bar{r}^{2}}+\left(\bar{e}_{1}+\bar{e}_{2}\right) \frac{1}{\bar{r}} \frac{d}{d \bar{r}}-a^{2} \bar{e}_{6} \frac{1}{\bar{r}^{2}}-b^{2} \bar{e}_{5}\right] \bar{u}_{n m i}} \\
-a\left[\left(\bar{e}_{2}+\bar{e}_{6}\right) \frac{1}{\bar{r}} \frac{d}{d \bar{r}}-\bar{e}_{6} \frac{1}{\bar{r}^{2}}\right] \bar{v}_{n m i}-b \bar{e}_{3} \frac{1}{\bar{r}} \bar{w}_{n m i} \\
-b\left(\bar{e}_{3}+\bar{e}_{5}\right) \frac{d \bar{w}_{n m i}}{d \bar{r}} \\
-\left[\bar{\eta}_{1}\left(\frac{d^{2}}{d \bar{r}^{2}}+\frac{1}{\bar{r}} \frac{d}{d \bar{r}}\right)-a^{2} \bar{\eta}_{2} \frac{1}{\bar{r}^{2}}-b^{2} \bar{\eta}_{3}\right] \bar{\phi}_{n m i} \\
+\bar{p}_{1}\left(\frac{d}{d \bar{r}}+\frac{1}{\bar{r}}\right) \bar{T}_{n m i}=0 .
\end{gathered}
$$

Furthermore, substituting the trigonometric series (15a) to $(15 \mathrm{e})$ into boundary and continuous conditions (10c) to (10e) and (11c) to (11f), we can obtain

$$
\begin{aligned}
& \bar{r}=\frac{r_{1}}{r_{0}}: \bar{T}_{n m 1}=\bar{T}_{n m a}, \\
& \bar{r}=\frac{r_{N+2}}{r_{0}}: \bar{T}_{n m, N+1}=\bar{T}_{n m b} \text {, } \\
& \bar{r}=\frac{r_{i+1}}{r_{0}}: \bar{T}_{n m i}=\bar{T}_{n m, i+1} \text {; } \\
& \bar{\lambda}_{i} \frac{d \bar{T}_{n m i}}{d \bar{r}}=\bar{\lambda}_{i+1} \frac{\partial \bar{T}_{n m, i+1}}{\partial \bar{r}} \\
& \bar{r}=\frac{r_{1}}{r_{0}}: \\
& \bar{C}_{111} \frac{d \bar{u}_{n m 1}}{d \bar{r}}+\bar{C}_{121} \frac{\bar{u}_{n m 1}}{\bar{r}}-a \bar{C}_{121} \frac{\bar{v}_{n m 1}}{\bar{r}} \\
& -b \bar{C}_{131} \bar{w}_{n m 1}-\bar{\beta}_{r 1} \bar{T}_{n m 1}=\bar{q}_{n m a} \\
& b \bar{u}_{n m 1}+\frac{d \bar{w}_{n m 1}}{d \bar{r}}=0 \\
& a \frac{\bar{u}_{n m 1}}{\bar{r}}+\frac{d \bar{v}_{n m 1}}{d \bar{r}}-\frac{\bar{v}_{n m 1}}{\bar{r}}=0 \\
& \bar{r}=\frac{r_{N+2}}{r_{0}}: \\
& \bar{\phi}_{n m, N+1}=0 \text {, } \\
& \bar{e}_{1} \frac{d \bar{u}_{n m i}}{d \bar{r}}+\frac{\bar{e}_{2}}{\bar{r}}\left(\bar{u}_{n m i}-a \bar{v}_{n m i}\right)-b \bar{e}_{3} \bar{w}_{n m i} \\
& -\bar{\eta}_{1} \frac{d \bar{\phi}_{n m i}}{d \bar{r}}+\bar{p}_{1} \bar{T}_{n m i}=0 \\
& \bar{C}_{11 i} \frac{d \bar{u}_{n m i}}{d \bar{r}}+\bar{C}_{12 i}\left(-\frac{a}{\bar{r}} \bar{v}_{n m i}+\bar{u}_{n m i}\right)-b \bar{C}_{13 i} \bar{w}_{n m i} \\
& -\bar{\beta}_{r i} \bar{T}_{n m i}+\bar{e}_{1} \frac{d \bar{\phi}_{n m i}}{d \bar{r}}=\bar{q}_{n m b}
\end{aligned}
$$




$$
\begin{aligned}
& \bar{C}_{66 i}\left(\frac{a}{\bar{r}} \bar{u}_{n m i}+\frac{d \bar{v}_{n m i}}{d \bar{r}}-\frac{\bar{v}_{n m i}}{\bar{r}}\right)+\frac{a \bar{e}_{6}}{\bar{r}} \bar{\phi}_{n m i}=0, \\
& \bar{C}_{55 i}\left(\frac{d \bar{w}_{n m i}}{d \bar{r}}+b \bar{u}_{n m i}\right)+b \bar{e}_{5} \bar{\phi}_{n m i}=0 \\
& \bar{r}=\frac{r_{i+1}}{r_{0}}: \\
& \bar{u}_{n m i}=\bar{u}_{n m, i+1}, \quad \bar{v}_{n m i}=\bar{v}_{n m, i+1}, \quad \bar{w}_{n m i}=\bar{w}_{n m, i+1} \\
& \bar{C}_{11 i} \frac{d \bar{u}_{n m i}}{d \bar{r}}+\bar{C}_{12 i}\left(-\frac{a}{\bar{r}} \bar{v}_{n m i}+\bar{u}_{n m i}\right) \\
& -b \bar{C}_{13 i} \bar{w}_{n m i}-\bar{\beta}_{r i} \bar{T}_{n m i}=\bar{C}_{11, i+1} \frac{d \bar{u}_{n m, i+1}}{d \bar{r}} \\
& +\bar{C}_{12, i+1}\left(-\frac{a}{\bar{r}} \bar{v}_{n m, i+1}+\bar{u}_{n m, i+1}\right) \\
& -b \bar{C}_{13, i+1} \bar{w}_{n m, i+1}-\bar{\beta}_{r, i+1} \bar{T}_{n m, i+1} \\
& \bar{C}_{66 i}\left(\frac{a}{\bar{r}} \bar{u}_{n m i}+\frac{d \bar{v}_{n m i}}{d \bar{r}}-\frac{\bar{v}_{n m i}}{\bar{r}}\right) \\
& =\bar{C}_{66, i+1}\left(\frac{a}{\bar{r}} \bar{u}_{n m, i+1}+\frac{d \bar{v}_{n m, i+1}}{d \bar{r}}-\frac{\bar{v}_{n m, i+1}}{\bar{r}}\right) \\
& \bar{C}_{55 i}\left(\frac{d \bar{w}_{n m i}}{d \bar{r}}+b \bar{u}_{n m i}\right) \\
& =\bar{C}_{55, i+1}\left(\frac{d \bar{w}_{n m, i+1}}{d \bar{r}}+b \bar{u}_{n m, i+1}\right) .
\end{aligned}
$$

Using the orthogonality of trigonometric functions, we can obtain

$$
\begin{aligned}
& \left\{\begin{array}{l}
\bar{T}_{n m a} \\
\bar{T}_{n m b} \\
\bar{q}_{n m a} \\
\bar{q}_{n m b}
\end{array}\right\} \\
& =\frac{4 r_{0}}{\theta_{0} l} \int_{0}^{\theta_{0}} \int_{0}^{l / r_{0}}\left\{\begin{array}{l}
\bar{T}_{a}(\theta, \bar{z}) \\
\bar{T}_{b}(\theta, \bar{z}) \\
\bar{q}_{a}(\theta, \bar{z}) \\
\bar{q}_{b}(\theta, \bar{z})
\end{array}\right\} \sin (a \theta) \sin (b \bar{z}) d \theta d \bar{z} .
\end{aligned}
$$

\section{Theoretical Analysis}

Based on the series solving method of ordinary differential equations, if the coefficient items of the ordinary differential equations were analytical at point $(\bar{r}-1)$ and could be expressed as Taylor's series of $(\bar{r}-1)$, the solutions of (17a)(17c) and (18) can also be expressed as the following Taylor's series [17]:

$$
\begin{array}{ll}
\bar{T}_{n m i}(\bar{r})=\sum_{k=1}^{\infty} A_{k i}(s)(\bar{r}-1)^{k} & i=1, N+1 \\
\bar{u}_{n m i}(\bar{r})=\sum_{k=1}^{\infty} B_{k i}(s)(\bar{r}-1)^{k} & i=1, N+1
\end{array}
$$

$$
\begin{array}{ll}
\bar{v}_{n m i}(\bar{r})=\sum_{k=1}^{\infty} C_{k i}(s)(\bar{r}-1)^{k} & i=1, N+1 \\
\bar{w}_{n m i}(\bar{r})=\sum_{k=1}^{\infty} D_{k i}(s)(\bar{r}-1)^{k} & i=1, N+1 \\
\bar{\phi}_{n m i}(\bar{r})=\sum_{k=1}^{\infty} F_{k i}(s)(\bar{r}-1)^{k} & i=N+1 .
\end{array}
$$

Substituting series (22) into (16) to (18), comparing the coefficient of $(\bar{r}-1)^{k}$, we can obtain the following recurrence equation:

$$
\begin{aligned}
& -(k+2)(k+1) A_{k+2, i} \\
& =(2 k+1)(k+1) A_{k+1, i}+k^{2} A_{k i}-\left(a^{2}+b^{2}\right) A_{k i} \\
& -2 b^{2} \frac{\bar{k}_{i}}{\overline{\bar{\lambda}}_{i}} A_{k-1, i}-b^{2} \frac{\bar{k}_{i}}{\overline{\bar{\lambda}}_{i}} A_{k-2, i} \\
& -(k+2)(k+1) B_{k+2, i} \\
& =(2 k+1)(k+1) B_{k+1, i} \\
& +\left(k^{2}-\frac{\bar{C}_{22 i}+b^{2} \bar{C}_{55 i}+a^{2} \bar{C}_{66 i}}{\bar{C}_{11 i}}\right) B_{k i}-2 b^{2} \frac{\bar{C}_{55 i}}{\bar{C}_{11 i}} B_{k-1, i} \\
& -b^{2} \frac{\bar{C}_{55 i}}{\bar{C}_{11 i}} B_{k-2, i}-a \frac{\left(\bar{C}_{12 i}+\bar{C}_{66 i}\right)}{\bar{C}_{11 i}}(k+1) C_{k+1, i} \\
& +a \frac{\left(\bar{C}_{22 i}+\bar{C}_{66 i}\right)}{\bar{C}_{11 i}} C_{k i}-a k \frac{\left(\bar{C}_{12 i}+\bar{C}_{66 i}\right)}{\bar{C}_{11 i}} C_{k i} \\
& -b \frac{\left(\bar{C}_{13 i}+\bar{C}_{55 i}\right)}{\bar{C}_{11 i}}(k+1) D_{k+1, i}-2 b \frac{k\left(\bar{C}_{13 i}+\bar{C}_{55 i}\right)}{\bar{C}_{11 i}} D_{k, i} \\
& -b \frac{\left(\bar{C}_{13 i}-\bar{C}_{23 i}\right)}{\bar{C}_{11 i}} D_{k, i}-\frac{\bar{\beta}_{r i}}{\bar{C}_{11 i}}(k+1) A_{k+1, i} \\
& -b\left[\frac{(k-1)\left(\bar{C}_{13 i}+\bar{C}_{55 i}\right)}{\bar{C}_{11 i}}+\frac{\left(\bar{C}_{13 i}-\bar{C}_{23 i}\right)}{\bar{C}_{11 i}}\right] D_{k-1, i} \\
& -\frac{(2 k+1) \bar{\beta}_{r i}-\bar{\beta}_{\theta i}}{\bar{C}_{11 i}} A_{k, i}-\frac{k \bar{\beta}_{r i}-\bar{\beta}_{\theta i}}{\bar{C}_{11 i}} A_{k-1, i} \\
& +(k+2)(k+1) \frac{\bar{e}_{1}}{\bar{C}_{11 i}} F_{k+2, i} \\
& +\frac{(k+1)\left[(2 k+1) \bar{e}_{1}-\bar{e}_{2}\right]}{\bar{C}_{11 i}} F_{k+1, i} \\
& +\frac{1}{\bar{C}_{11 i}}\left(k^{2} \bar{e}_{1}-k \bar{e}_{2}-a^{2} \bar{e}_{6}\right) F_{k, i} \\
& -b^{2} \frac{\bar{e}_{5}}{\bar{C}_{11 i}} F_{k, i}-b^{2} \frac{\bar{e}_{5}}{\bar{C}_{11 i}}\left(2 F_{k-1, i}+F_{k-2, i}\right)
\end{aligned}
$$




$$
\begin{aligned}
-(k+2)(k+1) C_{k+2, i} & \\
= & (2 k+1)(k+1) C_{k+1, i} \\
& +\left(k^{2}-\frac{a^{2} \bar{C}_{22 i}+b^{2} \bar{C}_{44 i}}{\bar{C}_{66 i}}-1\right) C_{k, i}-2 \frac{b^{2} \bar{C}_{44 i}}{\bar{C}_{66 i}} C_{k-1, i} \\
& -\frac{b^{2} \bar{C}_{44 i}}{\bar{C}_{66 i}} C_{k-2, i}+a \frac{\bar{C}_{12 i}+\bar{C}_{66 i}}{\bar{C}_{66 i}}(k+1) B_{k+1, i} \\
& +a k \frac{\bar{C}_{12 i}+\bar{C}_{66 i}}{\bar{C}_{66 i}} B_{k, i}+a \frac{\bar{C}_{22 i}+\bar{C}_{66 i}}{\bar{C}_{66 i}} B_{k, i} \\
& -a b \frac{\bar{C}_{23 i}+\bar{C}_{44 i}}{\bar{C}_{66 i}}\left(D_{k i}+D_{k-1, i}\right)-\frac{a \bar{\beta}_{\theta i}}{\bar{C}_{66 i}}\left(A_{k i}+A_{k-1, i}\right) \\
& -a \frac{\bar{e}_{2}+\bar{e}_{6}}{\bar{C}_{66 i}}\left[(k+1) F_{k+1, i}+k F_{k i}\right]
\end{aligned}
$$

$$
\begin{aligned}
-(k+2)(k+1) D_{k+2, i} & \\
= & (2 k+1)(k+1) D_{k+1, i} \\
& +\left(k^{2}-\frac{a^{2} \bar{C}_{44 i}+b^{2} \bar{C}_{33 i}}{\bar{C}_{55 i}}\right) D_{k i}-2 b^{2} \frac{\bar{C}_{33 i}}{\bar{C}_{55 i}} D_{k-1, i} \\
& -b^{2} \frac{\bar{C}_{33 i}}{\bar{C}_{55 i}} D_{k-2, i}+b \frac{\left(\bar{C}_{13 i}+\bar{C}_{55 i}\right)}{\bar{C}_{55 i}}(k+1) B_{k+1, i} \\
& +\frac{b}{\bar{C}_{55 i}}\left[2 k\left(\bar{C}_{13 i}+\bar{C}_{55 i}\right)+\left(\bar{C}_{23 i}+\bar{C}_{55 i}\right)\right] B_{k, i} \\
& -a b \frac{\left(\bar{C}_{23 i}+\bar{C}_{44 i}\right)}{\bar{C}_{55 i}}\left(C_{k, i}+C_{k-1, i}\right) \\
& -b \frac{\bar{\beta}_{z i}}{\bar{C}_{55 i}}\left(A_{k, i}+2 A_{k-1, i}+A_{k-2, i}\right)+\frac{(2 k+1) b \bar{e}_{5}}{\bar{C}_{55 i}} F_{k, i} \\
& +2 b k \frac{\bar{e}_{3}}{\bar{C}_{55 i}} F_{k, i}+\frac{b}{\bar{C}_{55 i}}\left[(k-1) \bar{e}_{3}+k \bar{e}_{5}\right] F_{k-1, i}
\end{aligned}
$$

$(k+2)(k+1) F_{k+2, i}$

$$
\begin{aligned}
= & -(2 k+1)(k+1) F_{k+1, i}-\left(k^{2}+\frac{a^{2} \bar{\eta}_{2}}{\bar{\eta}_{1}}+\frac{b^{2} \bar{\eta}_{3}}{\bar{\eta}_{1}}\right) F_{k i} \\
& +\frac{b^{2} \bar{\eta}_{3}}{\bar{\eta}_{1}}\left(2 F_{k-1, i}+F_{k-2, i}\right) \\
& +\frac{\bar{p}_{1}}{\bar{\eta}_{1}}\left[(k+1) A_{k+1, i}+(2 k+1) A_{k, i}+k A_{k-1, i}\right] \\
& +\frac{\bar{e}_{1}}{\bar{\eta}_{1}}(k+2)(k+1) B_{k+2, i}+\frac{\bar{e}_{1}}{\bar{\eta}_{1}}(2 k+1)(k+1) B_{k+1, i} \\
& +\frac{\bar{e}_{2}}{\bar{\eta}_{1}}(k+1) B_{k+1, i}+\frac{1}{\bar{\eta}_{1}}\left(\bar{e}_{1} k^{2}+\bar{e}_{2} k-a^{2} \bar{e}_{6}-b^{2} \bar{e}_{5}\right) B_{k, i}
\end{aligned}
$$

$$
\begin{aligned}
& -2 \frac{b^{2} \bar{e}_{5}}{\bar{\eta}_{1}} B_{k-1, i}-a \frac{\left(\bar{e}_{2}+\bar{e}_{6}\right)}{\bar{\eta}_{1}}(k+1) C_{k+1, i} \\
& -\frac{b^{2} \bar{e}_{5}}{\bar{\eta}_{1}} B_{k-2, i}-\frac{a \bar{e}_{2} k+a \bar{e}_{6}(k-1)}{\bar{\eta}_{1}} C_{k, i} \\
& -b \frac{\left(\bar{e}_{3}+\bar{e}_{5}\right)}{\bar{\eta}_{1}}(k+1) D_{k+1, i}-\frac{1}{\bar{\eta}_{1}} b \bar{e}_{3}(2 k+1) D_{k, i} \\
& -\frac{2 b \bar{e}_{5} k}{\bar{\eta}_{1}} D_{k, i}-\frac{b k \bar{e}_{3}}{\bar{\eta}_{1}} D_{k-1, i}-\frac{b(k-1) \bar{e}_{5}}{\bar{\eta}_{1}} D_{k-1, i},
\end{aligned}
$$

where $A_{-1, i}=A_{-2, i}=0$. From the recurrence equations (23) and (24a) to (24d), we can see that all coefficients $A_{k i}, B_{k i}, C_{k i}$, $D_{k i}$, and $F_{k i}$ in Taylor's series can be briefly expressed by $A_{o i}$, $A_{1 i}, B_{o i}, B_{1 i} ; C_{o i}, C_{1 i} ; D_{o i}, D_{1 i} ; F_{o i}$, and $F_{1 i}$ :

$$
\begin{aligned}
& A_{k i}=\xi_{1 i}^{k} A_{0 i}+\xi_{2 i}^{k} A_{1 i} ; \\
& B_{k i}= \zeta_{1 i}^{k} B_{0 i}+\zeta_{2 i}^{k} B_{1 i}+\zeta_{3 i}^{k} C_{0 i}+\zeta_{4 i}^{k} C_{1 i} \\
&+ \zeta_{5 i}^{k} D_{0 i}+\zeta_{6 i}^{k} D_{1 i}+\zeta_{7 i}^{k} F_{0 i}+\zeta_{8 i}^{k} F_{1 i} \\
&+ \zeta_{9 i}^{k} A_{0 i}+\zeta_{10 i}^{k} A_{1 i}, \\
& C_{k i}= L_{1 i}^{k} B_{0 i}+L_{2 i}^{k} B_{1 i}+L_{3 i}^{k} C_{0 i} \\
&+ L_{4 i}^{k} C_{1 i}+L_{5 i}^{k} D_{0 i}+L_{6 i}^{k} D_{1 i}+L_{7 i}^{k} F_{0 i} \\
&+ L_{8 i}^{k} F_{1 i}+L_{9 i}^{k} A_{0 i}+L_{10 i}^{k} A_{1 i}, \\
& D_{k i}=\rho_{1 i}^{k} B_{0 i}+\rho_{2 i}^{k} B_{1 i}+\rho_{3 i}^{k} C_{0 i} \\
&+\rho_{4 i}^{k} C_{1 i}+\rho_{5 i}^{k} D_{0 i}+\rho_{6 i}^{k} D_{1 i}+\rho_{7 i}^{k} F_{0 i} \\
&+\rho_{8 i}^{k} F_{1 i}+\rho_{9 i}^{k} A_{0 i}+\rho_{10 i}^{k} A_{1 i}, \\
& F_{k i}=\gamma_{1 i}^{k} B_{0 i}+\gamma_{2 i}^{k} B_{1 i}+\gamma_{3 i}^{k} C_{0 i}+\gamma_{4 i}^{k} C_{1 i} \\
&+\gamma_{5 i}^{k} D_{0 i}+\gamma_{6 i}^{k} D_{1 i}+\gamma_{7 i}^{k} F_{0 i}+\gamma_{8 i}^{k} F_{1 i} \\
&+\gamma_{9 i}^{k} A_{0 i}+\gamma_{10 i}^{k} A_{1 i},
\end{aligned}
$$

where the coefficients $\xi_{1 i}^{k}, \xi_{2 i}^{k} ; \zeta_{1 i}^{k} \sim \zeta_{10 i}^{k} ; L_{1 i}^{k} \sim L_{10 i}^{k} ; \rho_{1 i}^{k} \sim$ $\rho_{10 i}^{k}$ and $\gamma_{1 i}^{k} \sim \gamma_{10 i}^{k}$ can be derived from the recurrence equations (23) and (24a) to (24d). Substituting the solved series coefficients (25a) to (25d) into Taylor series (22), we can obtain $\bar{T}_{n m i}(\bar{r}), \bar{u}_{n m i}(\bar{r}), \bar{v}_{n m i}(\bar{r}), \bar{w}_{n m i}(\bar{r})$ and $\bar{\phi}_{n m i}(\bar{r})$.

Where $A_{0 i}, A_{1 i} ; B_{0 i}, B_{1 i} ; C_{0 i}, C_{1 i} ; D_{0 i}, D_{1 i} ; F_{0 i}$ and $F_{1 i}$ are unknown constants and they can be determined by substituting solutions (24a)-(24d) into the boundary and continuous conditions (19a) to (19c) and (20a) to (20c).

Substituting solutions $\bar{T}_{n m i}(\bar{r}), \bar{u}_{n m i}(\bar{r}), \bar{v}_{n m i}(\bar{r}), \bar{w}_{n m i}(\bar{r})$, and $\bar{\phi}_{n m i}(\bar{r})$ into trigonometric series $(15 \mathrm{a})-(15 \mathrm{e})$, we can obtain the steady-state piezothermomechanical solution for the finite cylindrical composite panel. Furthermore, using geometric equation and constitutive equation, the analytical solutions of thermo-mechanical stresses for the laminated cylinder can be obtained. 


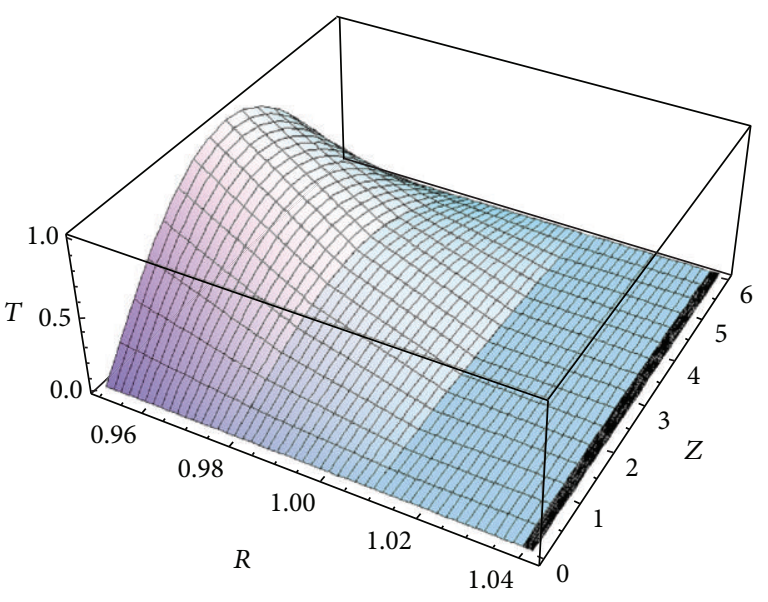

(a)

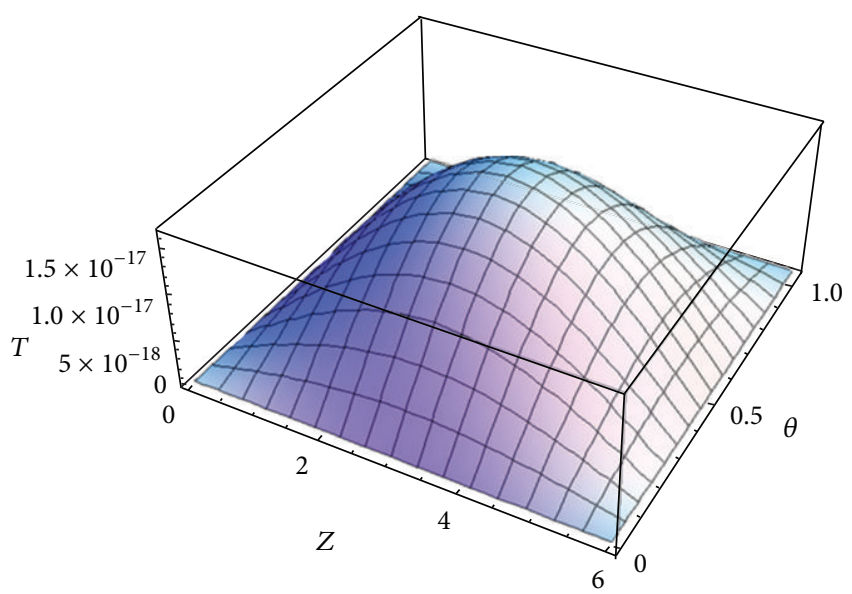

(b)

Figure 2: Dimensionless temperature distribution: (a) at $\operatorname{section} \theta=$ $\pi / 6$, (b) $R=1.042$.

\section{Numerical Results and Discussion}

To illustrate the above analysis, we investigate the piezothermoelastic stresses in a functionally graded cylindrical shell with a piezoelectric layer on outside. The dimensions $L=6$, $\theta_{0}=\pi / 3, R_{a}=0.95$, and $R_{b}=1.042$, the thickness of piezoelectric layer is 0.002 , the thickness of each layer is assumed to be equivalent. The reference values of temperature, Young's modulus, and thermal expansion coefficient are $T_{0}=200 \mathrm{~K}$, $E_{0}=300 \mathrm{GPa}$, and $\alpha_{0}=4.9 \times 10^{-5} \mathrm{~K}^{-1}$, respectively.

In this paper, the functionally graded finite cylinder made of mullite and molybdenum is analyzed. The thermoelastic properties of mullite and molybdenum are given as follows $[11,19]$ :

$$
\begin{aligned}
& E_{m}=330 \mathrm{GPa}, \quad \mu_{m}=0.3 ; \quad \lambda_{m}=138 \mathrm{~W} / \mathrm{mK} ; \\
& \alpha_{m}=4.9 \times 10^{-6} / \mathrm{K} \\
& E_{c}=330 \mathrm{GPa}, \quad \mu_{c}=0.3 ; \quad \lambda_{c}=138 \mathrm{~W} / \mathrm{mK} ; \\
& \alpha_{c}=4.9 \times 10^{-6} / \mathrm{K} .
\end{aligned}
$$

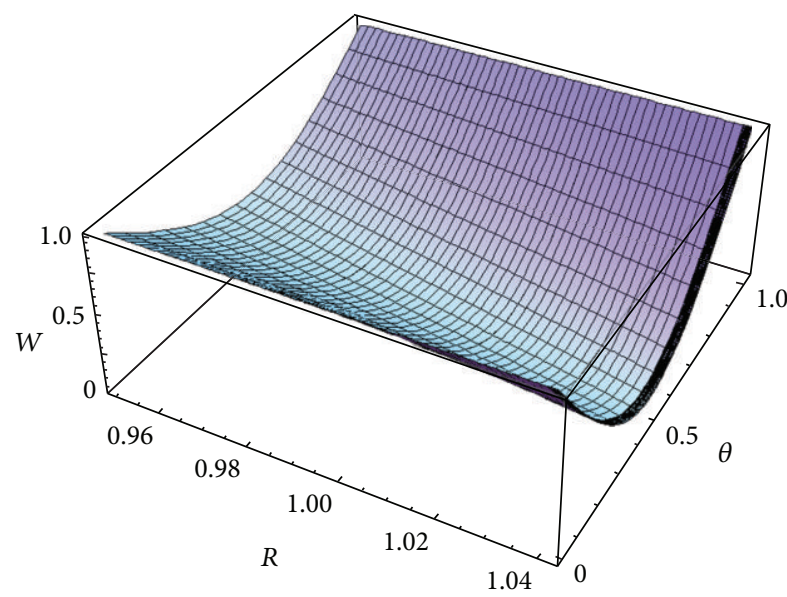

(a)

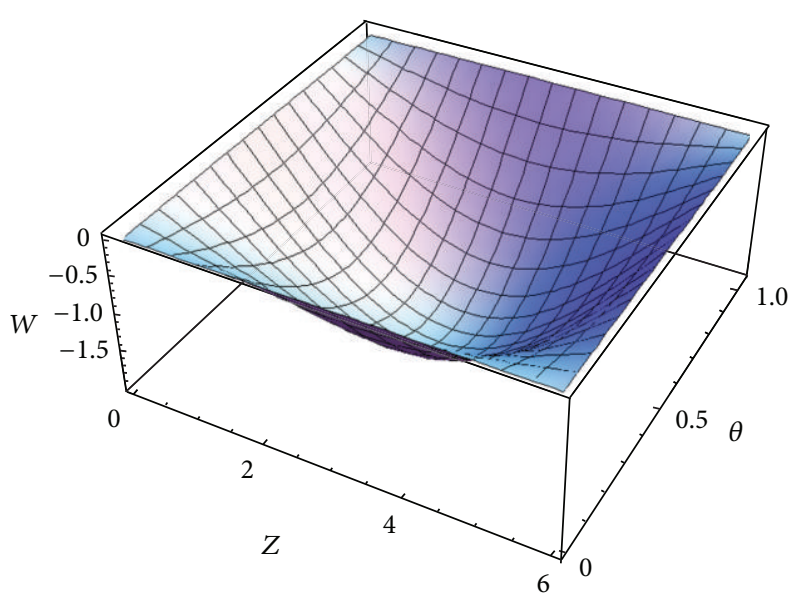

(b)

Figure 3: Dimensionless axial displacement distribution: (a) at section $\theta=\pi / 6$, (b) $R=1.042$.

The material properties of piezoelectric layer (cadmium selenite) are summarized as

$$
\begin{gathered}
C_{11}=83.6 \mathrm{GPa}, \quad C_{22}=C_{33}=74.1 \mathrm{GPa} \\
C_{23}=45.2 \mathrm{GPa}, \quad C_{12}=C_{13}=39.3 \mathrm{GPa}, \\
C_{66}=13.17 \mathrm{GPa}, \quad e_{1}=0.347 \mathrm{C} / \mathrm{m}^{2}, \\
e_{2}=e_{3}=-0.16 \mathrm{C} / \mathrm{m}^{2}, \quad e_{5}=e_{6}=-0.138 \mathrm{C} / \mathrm{m}^{2}, \\
\eta_{1}=9.03 \times 10^{-11} \mathrm{C}^{2} / \mathrm{Nm}^{2}, \\
\eta_{2}=\eta_{3}=8.25 \times 10^{-11} \mathrm{C}^{2} / \mathrm{Nm}^{2}, \\
P_{1}=-2.94 \times 10^{-6} \mathrm{C} / \mathrm{m}^{2} \mathrm{~K}, \\
d_{1}=-3.92 \times 10^{-12} \mathrm{C} / \mathrm{N}, \quad \lambda=8.6 \mathrm{~W} / \mathrm{mK} .
\end{gathered}
$$




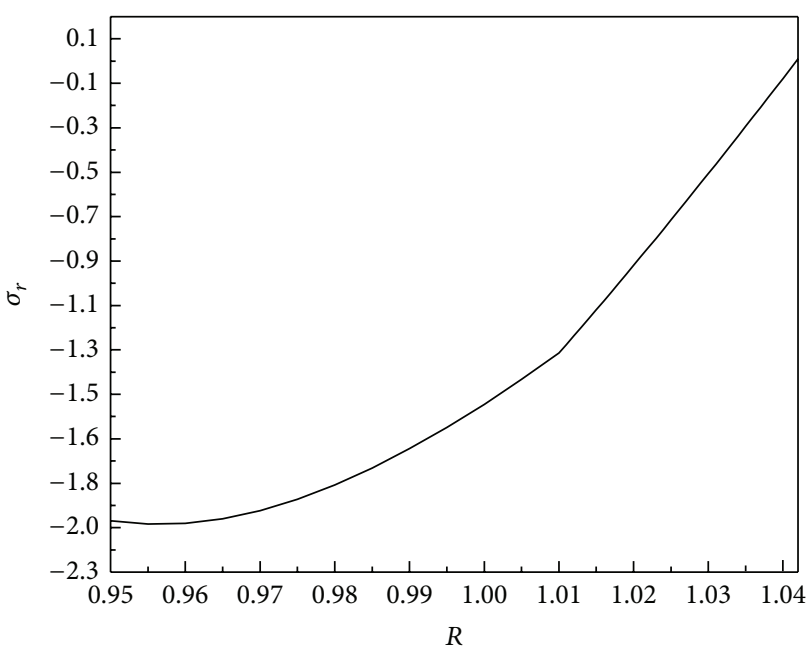

(a)

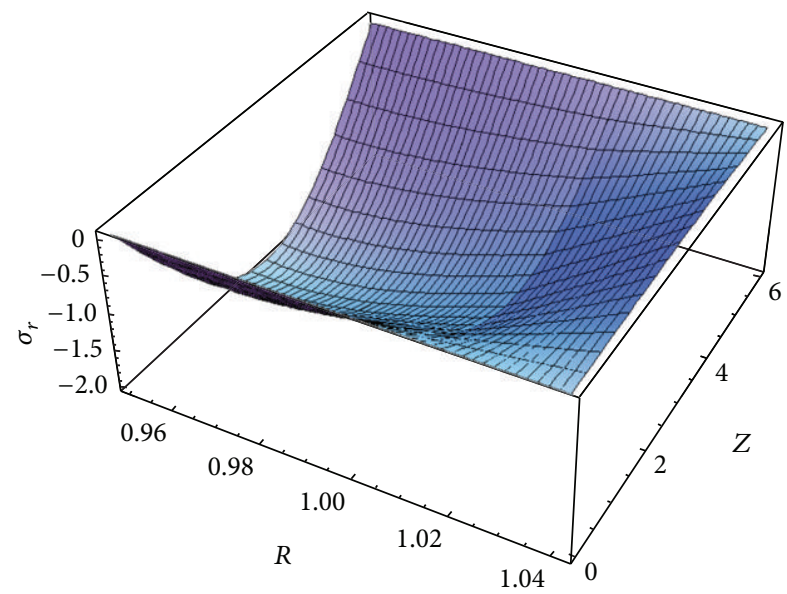

(b)

Figure 4: Distribution of dimensionless radial stress: (a) $Z=3.0$, $\theta=\pi / 6$, (b) $\theta=\pi / 6$.

And it is assumed that the cylindrical shell is subjected to the following nonumiform thermal and mechanical loadings on its outer surfaces:

$$
\begin{gathered}
T_{a}(\theta, z)=0 \quad \text { at } R_{a}=0.95 \\
T_{b}(\theta, z)=200 \times \sin [3 \theta] \times \sin \left[\frac{\pi z}{6}\right] \quad \text { at } R_{b}=1.04 \\
q_{a}(\theta, z)=0 \quad \text { at } R_{a}=0.95 \\
q_{b}(\theta, z)=-10^{8} \times \sin [3 \theta] \times \sin \left[\frac{\pi z}{6}\right] \quad \text { at } R_{b}=1.042 .
\end{gathered}
$$

Figures 2 to 8 illustrate the numerical results of dimensionless temperature, displacement, and stresses of functionally graded cylindrical shell with piezoelectric layer.

Figure 2 shows the numerical results of dimensionless temperature distributions. Due to the graded variation of the material properties along the radial direction, the temperature reduced in a nonlinear form through thickness direction.

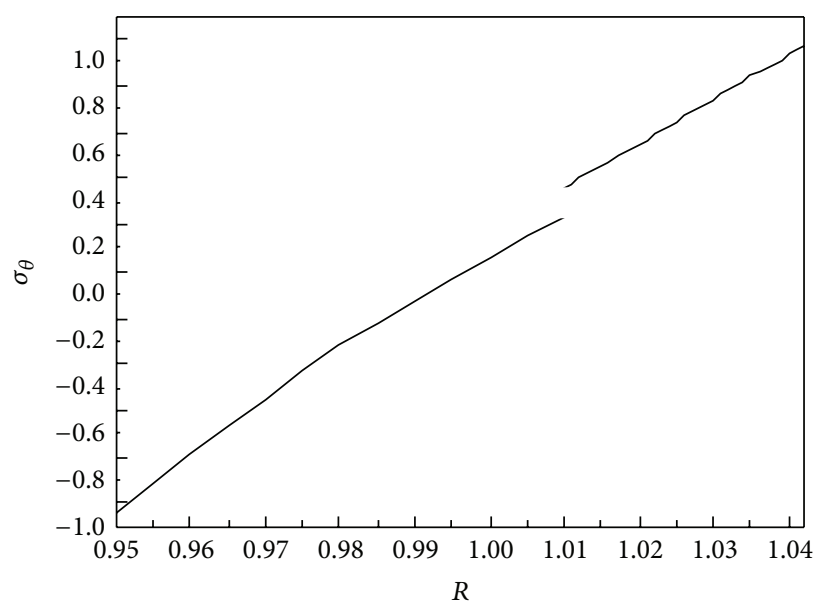

(a)

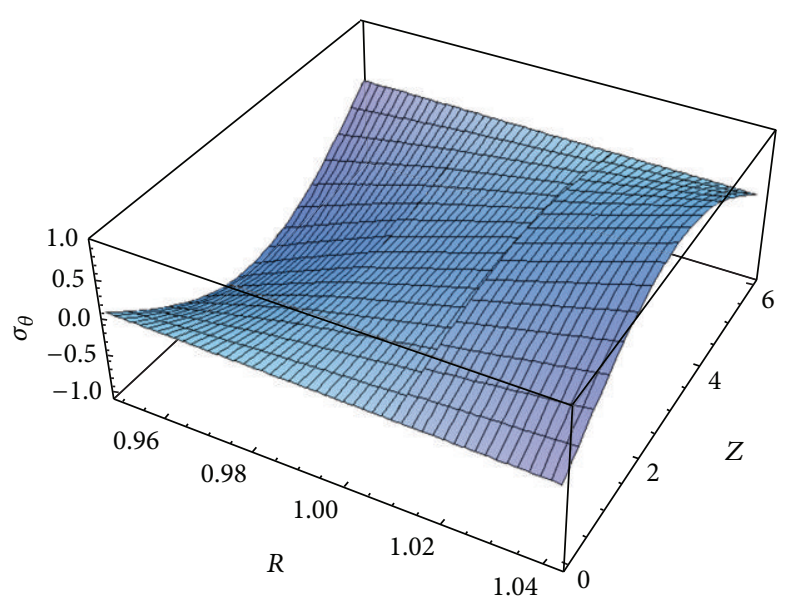

(b)

FIGURE 5: Distribution of dimensionless circumferential stress: (a) $Z=3.0, \theta=\pi / 6$, (b) $\theta=\pi / 6$.

For the thermal load is a function of sin, the maximum temperature in each layer is located along the axial $z=3$.

Figure 3 shows the axial displacement; the magnitude of axial displacement becomes larger near the outer surface. Figures 4 to 12 show the numerical results of dimensionless radial, hoop, axial, and shear stresses, respectively. The radial stress increases from the inner surface to outer surface nonlinearly; the radial stress is compressive for the inner heating and pressure loads.

Figure 5(a) shows the variation of circumferential stress on the thickness direction at point $Z=3.0, \theta=\pi / 6$. Due to the assumed thermomechanical loadings, the circumferential stress is compressive stress at the inner surface and is tensile stress at the outer surface. Because of the mismatch material properties, the circumferential stress is not continuous across the interfaces. Due to the assumed thermomechanical loading, the distribution of circumferential stress is nonuniform. It can be seen that maximum values of the circumferential stress occur at the same surface where the temperature loadings are applied. Similar distributions of circumferential stress can be obtained at other sections in the thickness 


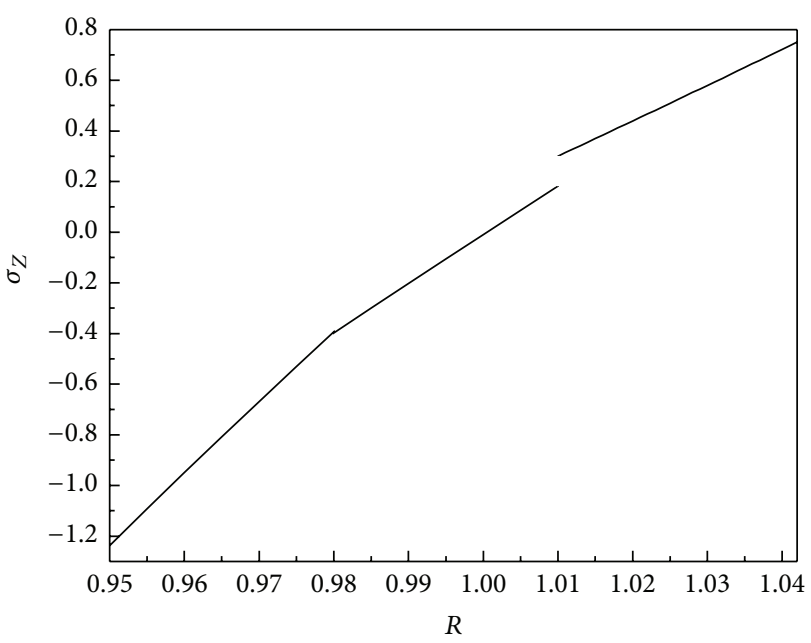

(a)

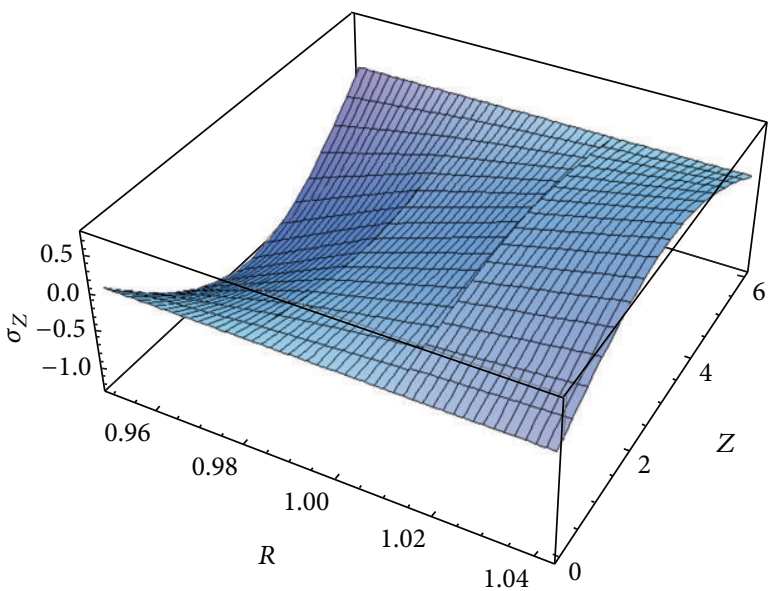

(b)

FIgURE 6: Distribution of dimensionless axial stress: (a) at point $Z=$ 3.0, $\theta=\pi / 6$. (b) $\theta=\pi / 6$.

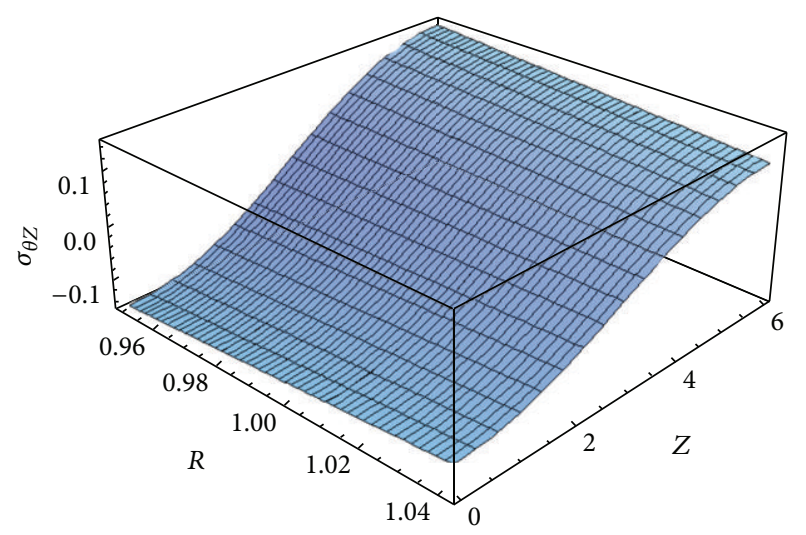

Figure 7: Distribution of dimensionless shear stress $\sigma_{\theta Z}(R=1.04)$.

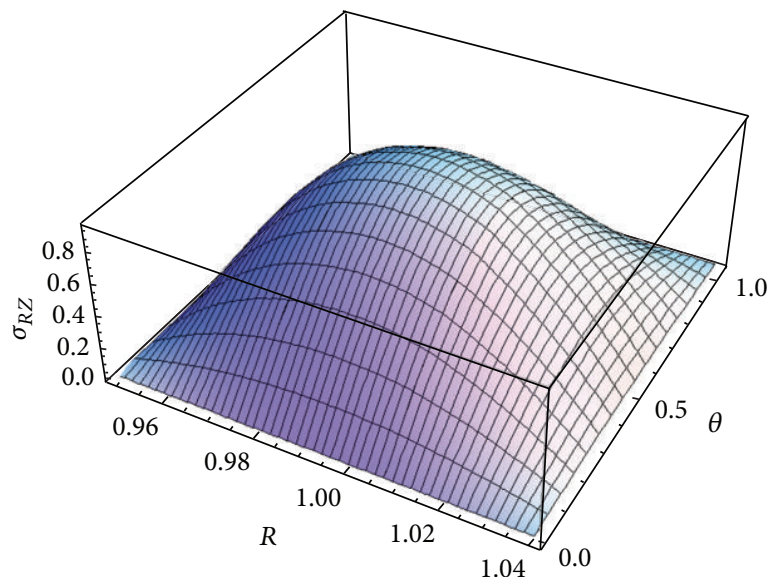

(a)

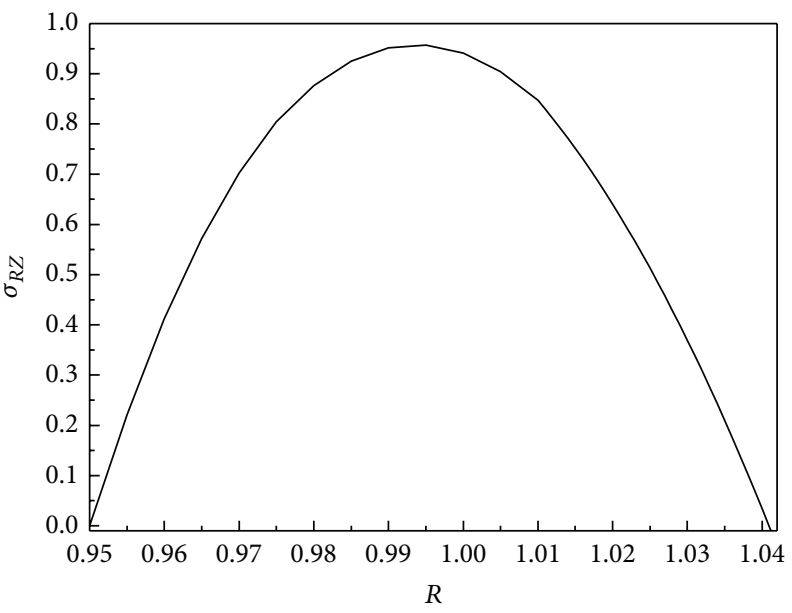

(b)

Figure 8: Distribution of dimensionless shear stress $\sigma_{R Z}$ : (a) $Z=$ 3.0, (b) at point $Z=3.0, \theta=\pi / 6$.

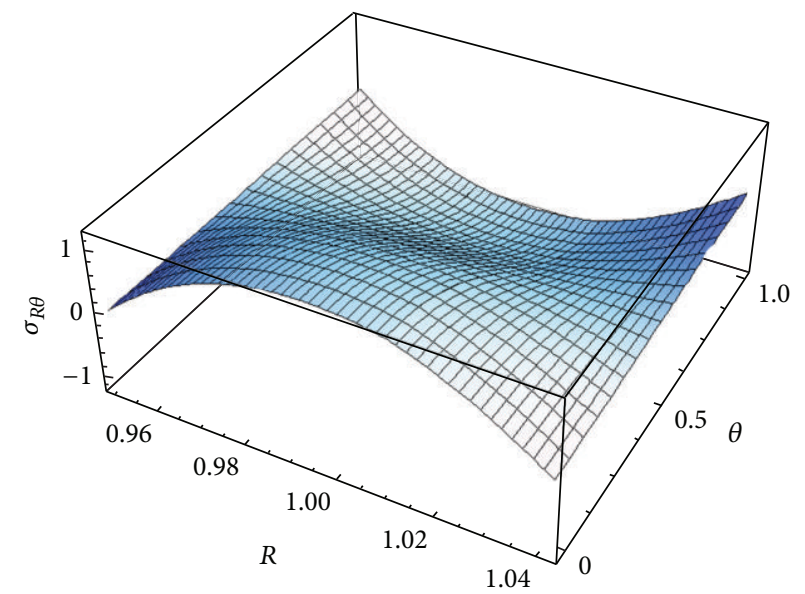

FIGURE 9: Distribution of dimensionless shear stress $(Z=3)$. 


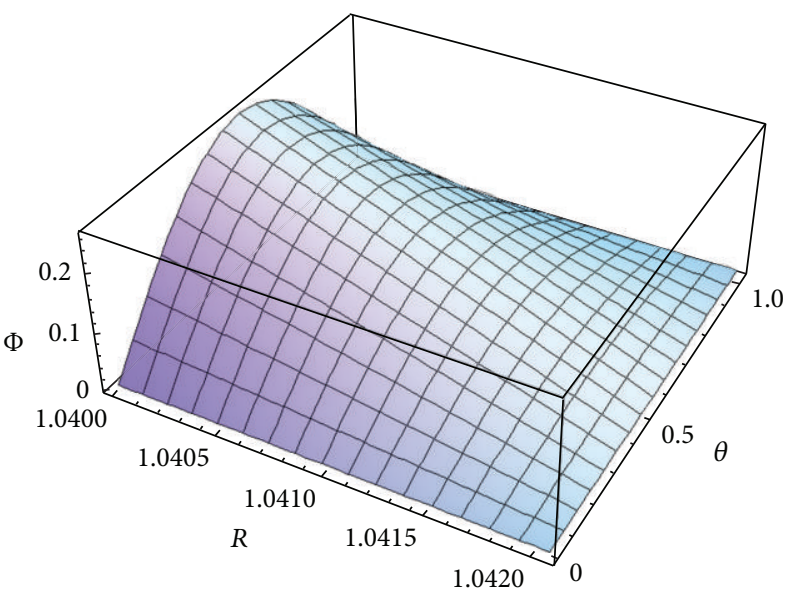

(a)

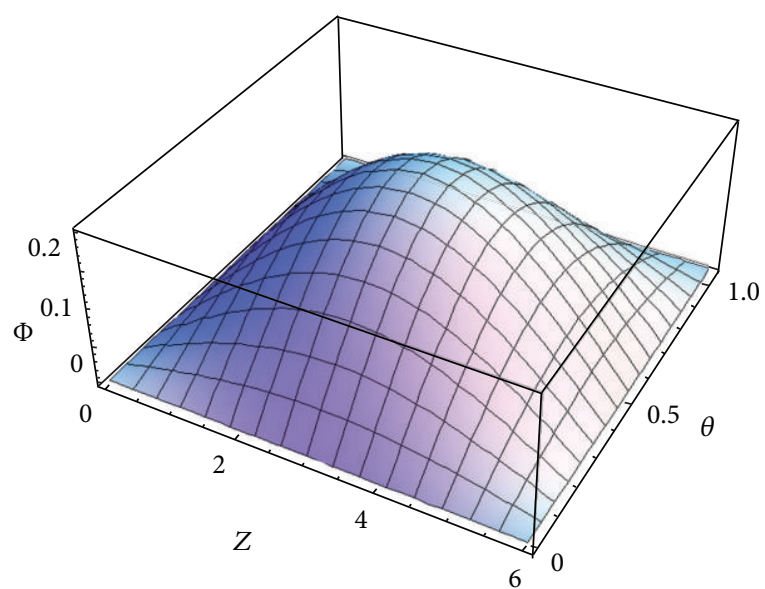

(b)

Figure 10: Distribution of dimensionless electric potential: (a) at section $Z=3$, (b) $R=1.04$.

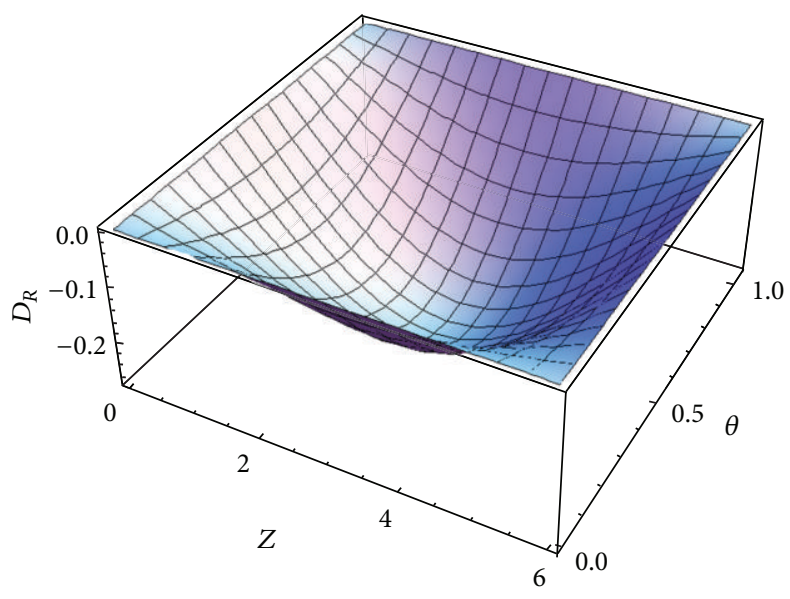

(a)

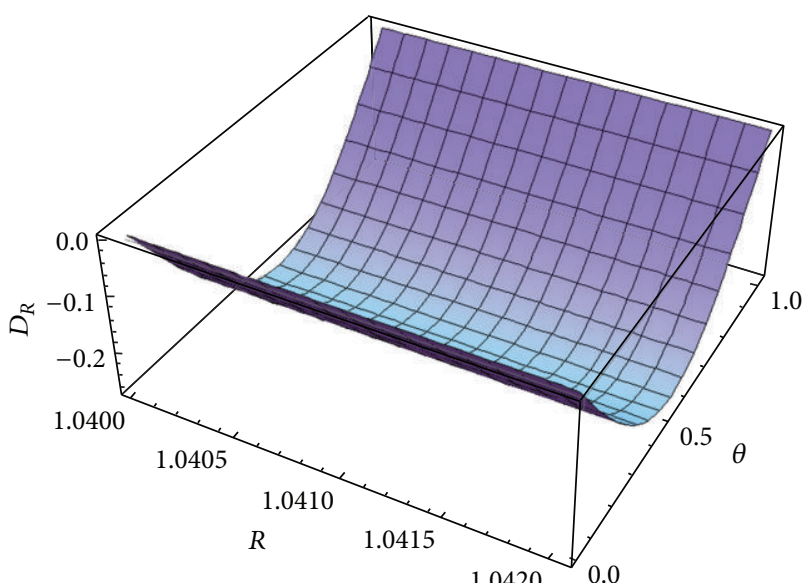

(b)

FIGURE 11: Distribution of dimensionless electric displacement $D_{R}$ : (a) at section $R=1.04$, (b) $Z=3$.

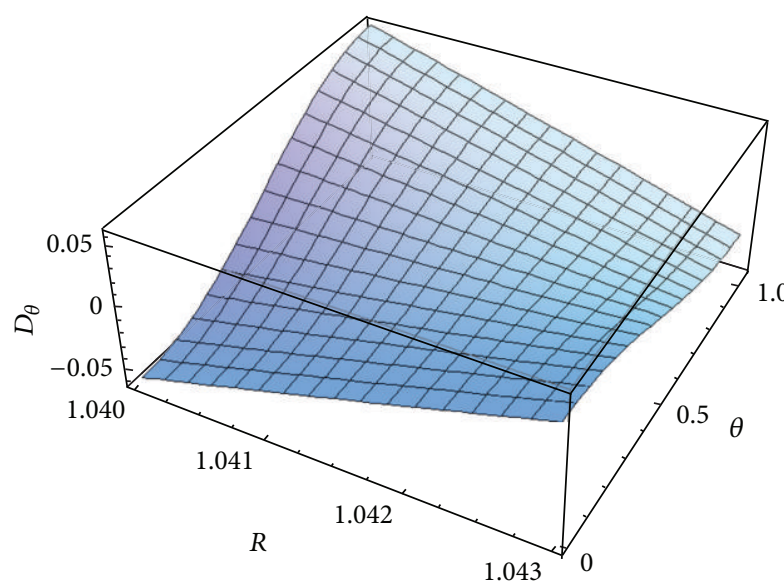

(a)

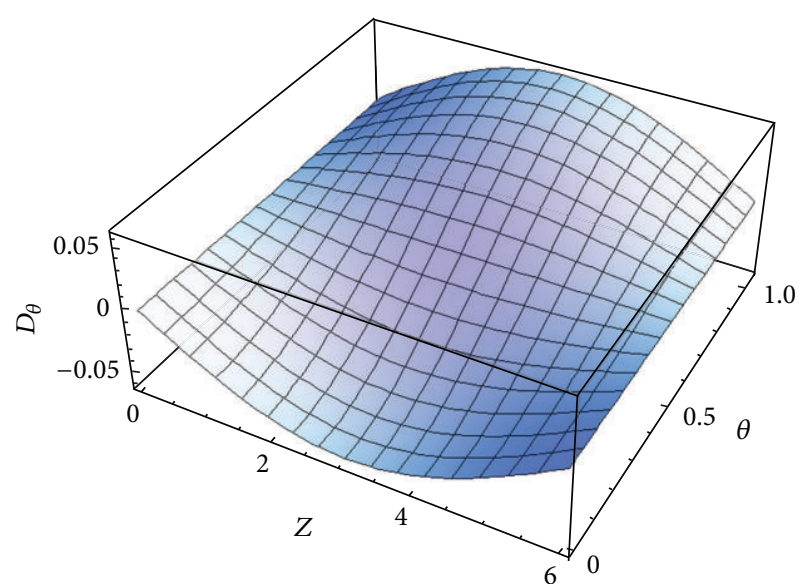

(b)

Figure 12: Distribution of dimensionless electric displacement $D_{\theta}$ : (a) at section $R=1.04$, (b) $Z=3$. 
direction. For the sake of brevity, the distributions in other thickness section are omitted here. Figure 6(a) shows the distributions of axial stress on the variation of thickness direction at $Z=3$ and $\theta=\pi / 6$. in Figure 6(b), due to the assumed boundary condition and thermomechanical loading, the axial stress is tensile stress at the inner surface and varies from compressive to tensile stress, which is similar to circumferential stress.

Figures 7 to 9 show the distributions of three shear stresses at different sections. It is seen that the maximum values of all three shear stresses are smaller than those of normal stresses. Due to the assumed thermo-mechanical loadings, distributions of the three shear stresses show different distribution. According to the boundary condition and material properties, the maximum shear stresses $\sigma_{R \theta}$ and $\sigma_{R Z}$ appear at the middle radius, while the shear stress $\sigma_{\theta Z}$ varies from compressive stress to tensile stress along its length. The maximum shear stress $\sigma_{R Z}$ appears at the middle along the length and the middle thickness. Figures 10, 11, and Figure 12 show the variation of electric potential and displacement along the thickness; the electric potential distribution is similar to the temperature distribution. The electric displacement varied nonlinearly.

\section{Conclusions}

The piezothermoelastic analysis for a functionally graded cylindrical shell with piezoelectric layer is presented in this paper. The cylindrical shell is simply supported at four end edges and subjected to thermomechanical loadings on its outer surfaces. The piezoelectric layers are polarized along radial direction as a sensor. An exact $3 \mathrm{D}$ analytical solution is derived by using multilayered approximate approach and series solving method. Referred to the presented solution, other temperature boundary conditions can be solved by suitable forms of series solutions to satisfy both temperature and simply supported boundary conditions. It is also worth noting that the trigonometric series, which are used to separate variables, are only suitable for simply supported at four end edges. For other supported boundary conditions, another suitable series form must be considered to satisfy the related boundary conditions. For different functionally graded material, this analytical method can also be used.

\section{Acknowledgment}

This project is supported by the National Natural Science Foundation of China (Grant nos. 51375396 and 11202164).

\section{References}

[1] I. F. PintoCarreia, C. M. MotaSoares, and C. A. MotaSoares, "Analysis of piezo-laminated axisymmetric shell: a semi analytical higher order model," in Computational Methods For Shell and Spatial Structures, pp. 1-19, IASS-IACM, 2000.

[2] M. Taya, A. A. Almajid, M. Dunn, and H. Takahashi, "Design of bimorph piezo-composite actuators with functionally graded microstructure," Sensors and Actuators A, vol. 107, no. 3, pp. 248-260, 2003.
[3] Y. Ootao and Y. Tanigawa, "Transient piezothermoelastic analysis for a functionally graded thermopiezoelectric hollow sphere," Composite Structures, vol. 81, no. 4, pp. 540-549, 2007.

[4] Y. Ootao and Y. Tanigawa, "Transient piezothermoelasticity for a cylindrical composite panel composed of cross-ply and piezoelectric laminae," International Journal of Mechanical Sciences, vol. 44, no. 9, pp. 1861-1877, 2002.

[5] Y. Ootao and Y. Tanigawa, "Transient piezothermoelasticity for a cylindrical composite panel composed of angle-ply and piezoelectric laminae," International Journal of Solids and Structures, vol. 39, no. 23, pp. 5737-5752, 2002.

[6] Y. Ootao and Y. Tanigawa, "Three-dimensional transient piezothermoelasticity in functionally graded rectangular plate bonded to a piezoelectric plate," International Journal of Solids and Structures, vol. 37, no. 32, pp. 4377-4401, 2000.

[7] Y. Ootao and Y. Tanigawa, "Three-dimensional transient piezothermoelasticity for a rectangular composite plate composed of cross-ply and piezoelectric laminae," International Journal of Engineering Science, vol. 38, no. 1, pp. 47-71, 2000.

[8] C.-Q. Chen and Y.-P. Shen, "Piezothermoelasticity analysis for a circular cylindrical shell under the state of axisymmetric deformation," International Journal of Engineering Science, vol. 34, no. 14, pp. 1585-1600, 1996.

[9] X.-H. Wu, Y.-P. Shen, and C. Chen, "An exact solution for functionally graded piezothermoelastic cylindrical shell as sensors or actuators," Materials Letters, vol. 57, no. 22-23, pp. 3532-3542, 2003.

[10] S. Kapuria and G. G. S. Achary, "A coupled consistent thirdorder theory for hybrid piezoelectric plates," Composite Structures, vol. 70, no. 1, pp. 120-133, 2005.

[11] Z. S. Shao, "Mechanical and thermal stresses of a functionally graded circular hollow cylinder with finite length," International Journal of Pressure Vessels and Piping, vol. 82, no. 3, pp. 155-163, 2005.

[12] D. J. Huang, H. J. Ding, and W. Q. Chen, "Piezoelasticity solutions for functionally graded piezoelectric beams," Smart Materials and Structures, vol. 16, no. 3, pp. 687-695, 2007.

[13] J. Yang and H. J. Xiang, "Thermo-electro-mechanical characteristics of functionally graded piezoelectric actuators," Smart Materials and Structures, vol. 16, no. 3, pp. 784-797, 2007.

[14] J. Ying and H. M. Wang, "Axisymmetric thermoelastic analysis in a finite hollow cylinder due to nonuniform thermal shock," International Journal of Pressure Vessels and Piping, vol. 87, no. 12, pp. 714-720, 2010.

[15] T. Roy, P. Manikandan, and D. Chakraborty, "Improved shell finite element for piezothermoelastic analysis of smart fiber reinforced composite structures," Finite Elements in Analysis and Design, vol. 87, no. 12, pp. 714-720, 2010.

[16] M. Zaman, Z. Yan, and L. Jiang, "Thermal effect on the bending behavior of curved functionally graded piezoelectric actuators," International Journal of Applied Mechanics, vol. 2, no. 4, pp. 787805, 2010.

[17] A. Alibeigloo, "Thermoelastic solution for static deformations of functionally graded cylindrical shell bonded to thin piezoelectric layers," Composite Structures, vol. 93, no. 2, pp. 961-972, 2011.

[18] R. A. Alashti and M. Khorsand, "Three-dimensional thermoelastic analysis of a functionally graded cylindrical shell with piezoelectric layers by differential quadrature method," International Journal of Pressure Vessels and Piping, vol. 88, no. 5-7, pp. 167-180, 2011. 
[19] M. Jabbari, S. Sohrabpour, and M. R. Eslami, "Mechanical and thermal stresses in a functionally graded hollow cylinder due to radially symmetric loads," International Journal of Pressure Vessels and Piping, vol. 79, no. 7, pp. 493-497, 2002. 


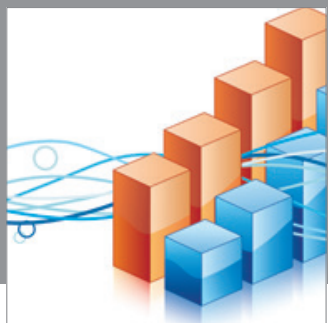

Advances in

Operations Research

mansans

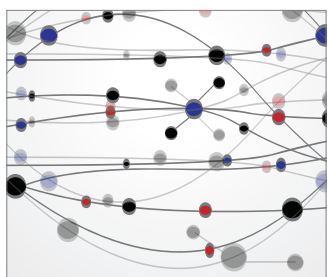

The Scientific World Journal
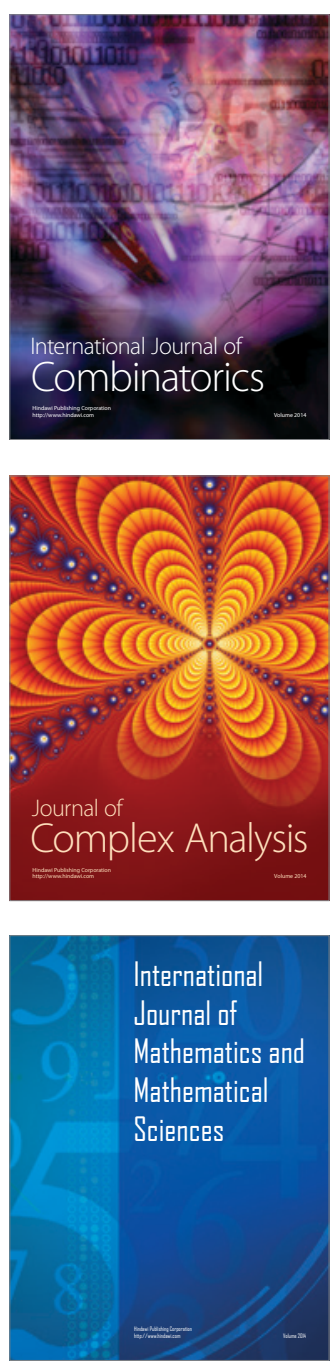
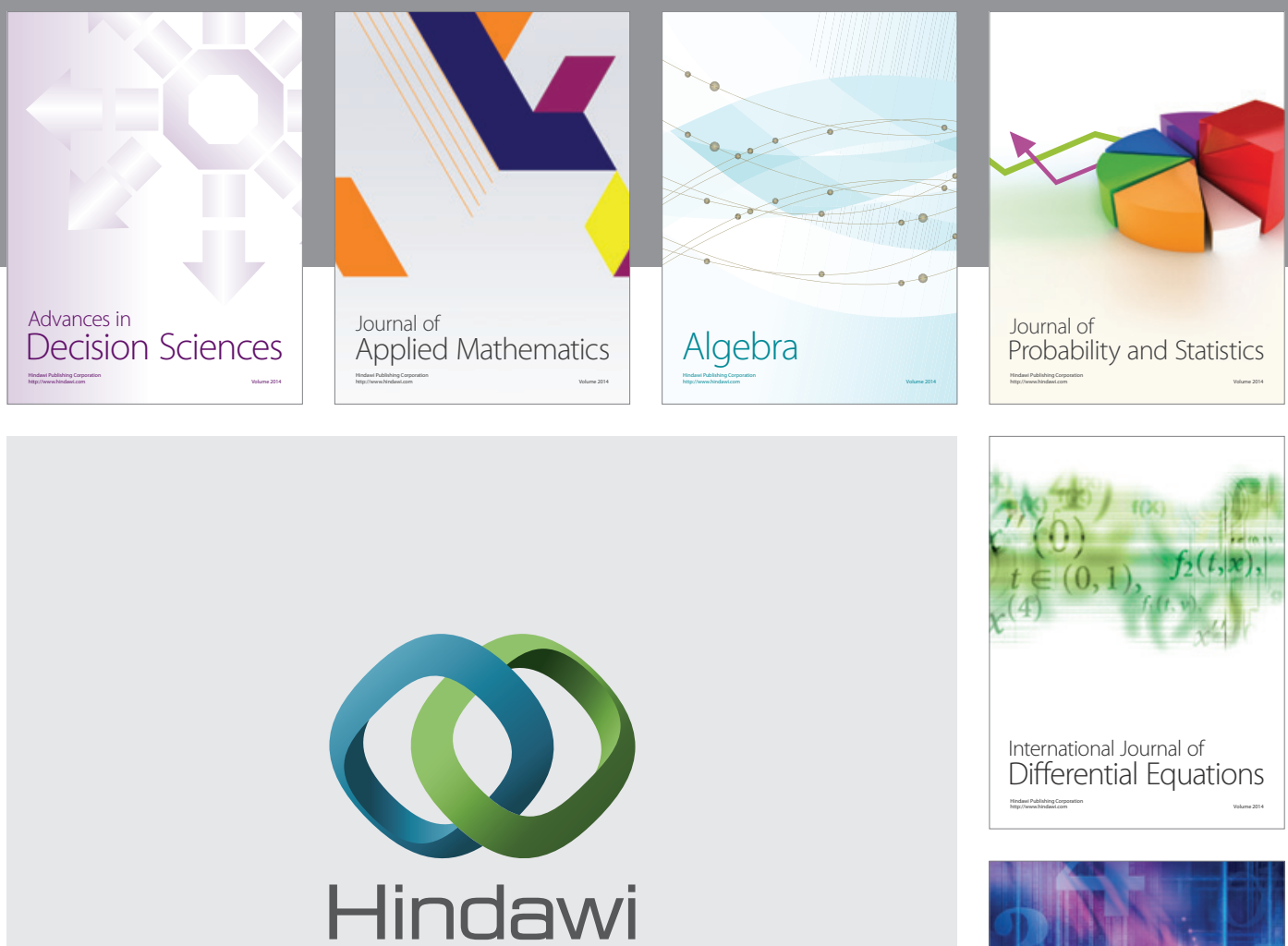

Submit your manuscripts at http://www.hindawi.com
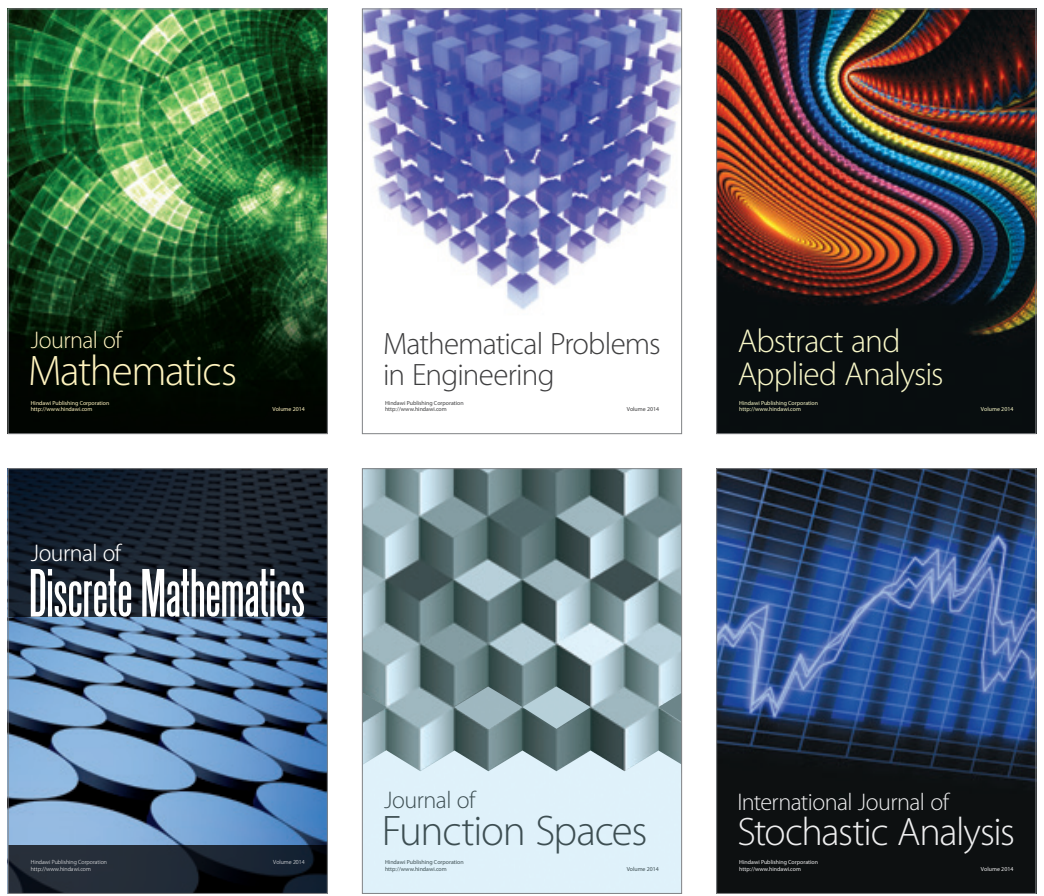

Journal of

Function Spaces

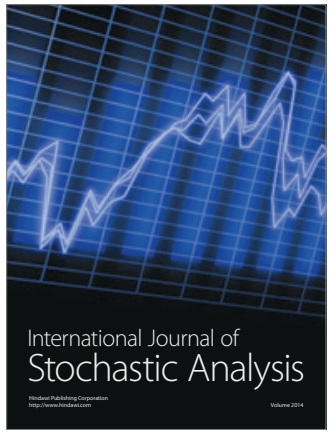

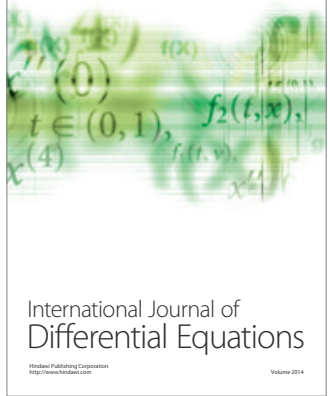
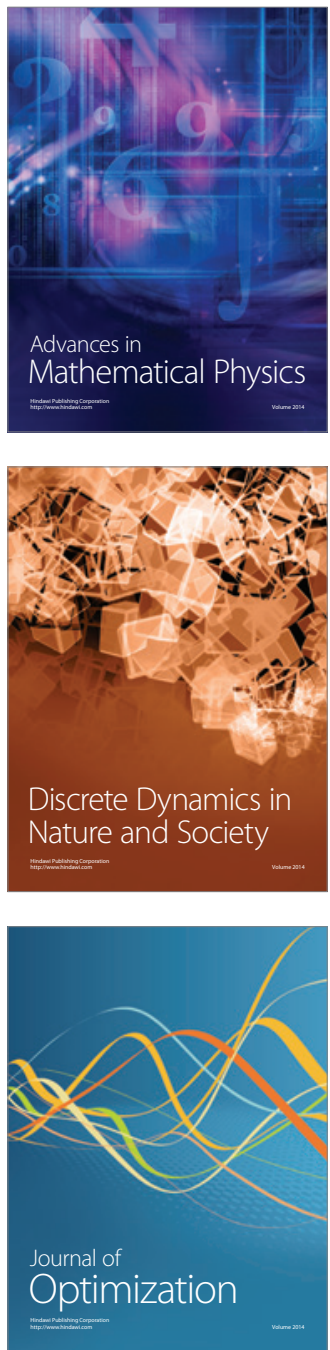